\title{
An expanded set of photoreceptors in the Eastern Pale Clouded Yellow butterfly, Colias erate
}

\author{
Primož Pirih · Kentaro Arikawa • Doekele G. Stavenga
}

Received: 16 May 2010 / Accepted: 17 May 2010 / Published online: 4 June 2010

(c) The Author(s) 2010. This article is published with open access at Springerlink.com

\begin{abstract}
We studied the spectral and polarisation sensitivities of photoreceptors of the butterfly Colias erate by using intracellular electrophysiological recordings and stimulation with light pulses. We developed a method of response waveform comparison (RWC) for evaluating the effective intensity of the light pulses. We identified one $\mathrm{UV}$, four violet-blue, two green and two red photoreceptor classes. We estimated the peak wavelengths of four rhodopsins to be at about 360, 420, 460 and $560 \mathrm{~nm}$. The four violet-blue classes are presumably based on combinations of two rhodopsins and a violet-absorbing screening pigment. The green classes have reduced sensitivity in the ultraviolet range. The two red classes have primary peaks at about 650 and $665 \mathrm{~nm}$, respectively, and secondary peaks at about $480 \mathrm{~nm}$. The shift of the main peak, so far the largest amongst insects, is presumably achieved by tuning the effective thickness of the red perirhabdomal screening pigment. Polarisation sensitivity of green and red photoreceptors is higher at the secondary than at the main peak. We found a 20 -fold variation of sensitivity within the cells of one green class, implying possible photoreceptor subfunctionalisation. We propose an allocation
\end{abstract}

P. Pirih $(\varangle) \cdot$ D. G. Stavenga

Department of Neurobiophysics,

University of Groningen, Groningen, The Netherlands

e-mail: p.pirih@rug.nl

P. Pirih

Department of Artificial Intelligence,

University of Groningen, Groningen, The Netherlands

K. Arikawa

Laboratory of Neuroethology,

Hayama Center for Advanced Studies,

Sokendai (The Graduate University for Advanced Studies),

Hayama, Kanagawa, Japan scheme of the receptor classes into the three ventral ommatidial types.

Keywords Vision · Rhodopsin · Colour · Lepidoptera . Spectral sensitivity

\section{Introduction}

The standard model for insect colour vision is a trichromatic system based on a set of ultraviolet (UV), blue (B), and green $(\mathrm{G})$ receptors, as in the honeybee (Menzel and Backhaus 1989; Land and Nilsson 2002) and also in some Lepidoptera (Hamdorf 1979; Kinoshita et al. 1997; White et al. 2003). The model is paralleled by the genetic analysis of opsins, where UV (peak wavelength, $\lambda_{\mathrm{p}}=330-380 \mathrm{~nm}$ ), violet/blue $\left(\lambda_{\mathrm{p}}=420-500 \mathrm{~nm}\right)$ and green $\left(\lambda_{\mathrm{p}}=500\right.$ $570 \mathrm{~nm}$ ) opsin clades have been identified (Briscoe 2008). Recent research has demonstrated that several butterfly species have diversified the spectral properties of the photoreceptors, by visual pigment gene duplication and/or applying spectral filtering, resulting in a visual system with up to eight different spectral classes (Arikawa et al. 1987; Briscoe and Chittka 2001; Sison-Mangus et al. 2006; Stavenga and Arikawa 2006; Briscoe 2008).

Each ommatidium in the butterfly compound eye contains nine photoreceptor cells, R1-R9 (Ribi 1978; Arikawa 1999). The light-sensing organelles of these photoreceptors, the rhabdomeres, are joined into a fused rhabdom, which acts as an optical waveguide. The rhabdomeres consist of microvilli containing the visual pigments, the rhodopsins. The absorption spectrum of the rhodopsin is the main determinant of a photoreceptor's spectral sensitivity, but distinct deviations can occur due to optical filtering, either resulting from light absorption by the other rhodopsins 
in the rhabdom or by screening pigments within or surrounding the light-guiding rhabdom (review Stavenga 2006).

The spectral organisation of the butterfly eye has been extensively studied in two species, the Japanese Yellow Swallowtail, Papilio xuthus, and the Small White, Pieris rapae crucivora. The localisation of the various photoreceptor types has been identified with a wide range of techniques, e.g., anatomy, molecular biology, optics and electrophysiology (Ribi 1978; Bandai et al. 1992; Arikawa and Uchiyama 1996; Arikawa and Stavenga 1997; Kitamoto et al. 2000; Qiu et al. 2002; Qiu and Arikawa 2003a; for review, see Arikawa 2003, Wakakuwa et al. 2007). To gain insight into the evolution of pierid butterflies (Stavenga and Arikawa 2006), we investigated the visual system of the eastern clouded yellow butterfly, Colias erate. So far, we have studied the molecular organisation (Awata et al. 2009) and ultrastructure of the eye of this species (Arikawa et al. 2009). Here, we report the spectral and polarisation sensitivities of photoreceptors of $C$. erate.

The compound eye of $C$. erate has a distinct dorsal area and a larger ventral area. The ommatidia in the ventral area contain a red screening pigment, which is concentrated in four clusters near the rhabdoms (Arikawa et al. 2009). The arrangement of the pigment clusters designates three ommatidial types. In the ommatidial types I, II and III, the clusters are arranged in a trapezoidal, square and rectangular manner, respectively. All ommatidia have a three-tiered fused rhabdom, where the distal and proximal tier consist of the rhabdomeres of photoreceptors R1-4 and R5-8, respectively; the very short basal tier contains the rhabdomere of photoreceptor R9.

There are four opsin-encoding mRNAs expressed in the retina of $C$. erate: $\mathrm{CeUV}, \mathrm{CeV} 1, \mathrm{CeV} 2$ and $\mathrm{CeL}$. The two sequence-wise similar opsins, $\mathrm{CeV} 1$ and $\mathrm{CeV} 2$, are always expressed together. In type I ommatidia, one of the distal R1 and R2 photoreceptors expresses CeUV, and the other coexpresses $\mathrm{CeV} 1$ and $\mathrm{CeV} 2$. In type II ommatidia, both $\mathrm{R} 1$ and $\mathrm{R} 2$ coexpress $\mathrm{CeV} 1$ and $\mathrm{CeV} 2$; in the type III ommatidia, both R1 and R2 express CeUV. The distal R3 and R4 as well as the proximal R5-R8 photoreceptors express CeL in all ommatidia (Awata et al. 2009).

Light that enters the eye is channelled by the dioptric system into the rhabdom, where it can be absorbed by the rhodopsins. Part of the light travels outside the rhabdom boundary; in the ventral eye area this light fraction can be absorbed by the red screening pigments. The light that escapes absorption reaches the proximal end of the rhabdom, a tracheolar tapetum, which acts as a multilayer reflector. The light reflected by the tapetum is reflected back into the rhabdom and has a second chance to be absorbed by the rhodopsins and screening pigment. Yet, a small light fraction remains unabsorbed, leaves the ommatidium and is observable as the eye shine (also called eye glow; Miller and Bernard 1968; Stavenga et al. 2001; Briscoe and Bernard 2005).

The eye shine phenomenon allows in vivo optical characterisation of individual ommatidia (Qiu et al. 2002; Vanhoutte et al. 2003). By performing imaging microspectrophotometry, we found that $C$. erate exhibits three classes of eye shine (Arikawa et al. 2009). The ommatidia in the dorsal eye area all have the same, rather bright red eye shine with a broad reflectance spectrum $(630-690 \mathrm{~nm}$, peak at $670 \mathrm{~nm}$ ). In the ventral eye area, the ommatidia with the so-called ventral red (VR) eye shine (spectral band from $650-690 \mathrm{~nm}$, peak at $670 \mathrm{~nm}$ ) correspond to ommatidial type I. These ommatidia additionally fluoresce in the green under violet excitation light (Arikawa et al. 2009). The fluorescent pigment presumably modifies the spectral sensitivity of distal photoreceptors, as in Pieris rapae males, where a receptor expressing a violet rhodopsin is modified into a double-peaked blue $(\mathrm{dB})$ receptor (Arikawa et al. 2005). The ommatidia with far red (FR) eye shine in the ventral area (spectral band from 690 to $750 \mathrm{~nm}$, peak at $730 \mathrm{~nm}$ ) correspond to ommatidial types II and III (Arikawa et al. 2009). The short-wavelength cutoff of the eye shine is due to the red screening pigment, which in addition presumably red-shifts the spectral sensitivity of the proximal photoreceptors, as in Pieris rapae (Qiu and Arikawa 2003b).

From the molecular, anatomical and optical evidence collected from $C$. erate, following the concepts revealed by previous work on Pieris and Papilio, we were expecting several types of modified short-wavelength photoreceptors and possibly two subclasses of red receptors with large sensitivity shifts. In the present study, we classified the photoreceptors of $C$. erate into nine classes by virtue of their spectral and polarisation sensitivities. We have developed a new method (response waveform comparison, RWC) for the estimation of effective intensities of light pulses, which is based on comparing the waveform of the voltage response with interpolated waveforms from the intensity-response calibration, and finding the most similar response waveform. We show that this method has distinct advantages over the more conventional measurement of response voltage amplitudes and estimation of effective intensities using the reverse transformation of the intensity-response curve. Additionally, we have measured the intensity-response characteristics of the measured photoreceptor classes. We discuss the mechanisms leading to the unusual spectral sensitivities of some of the photoreceptor classes, the function of red receptors and the evolution of multiple photoreceptor classes in butterflies. 


\section{Materials and methods}

\section{Experimental animals and preparation}

The experiments were performed on butterflies caught around the Sokendai-Hayama Campus, Shōnan Village, Kanagawa Prefecture, Japan. The butterflies were kept for up to 10 days in the laboratory and fed daily with sucrose solution.

\section{Light stimulation and electrophysiological recording}

We used a computer-controlled light stimulator consisting of a 500-W XBO arc lamp source, a shutter, four quartz neutral density filters with optical densities 1, 2, 3 and 4, respectively, a quartz grey wedge covering $2 \log$ units and a wheel with 23 interference filters [Asahi Spectra, full width at half maximum (FWHM) $10 \mathrm{~nm}$ ] going from 300 to $740 \mathrm{~nm}$ in 20-nm steps. The light beam was focused on one end of a quartz optical fibre, the other end of which was clamped on a perimeter device. The latter end was at a distance of $\sim 10 \mathrm{~cm}$ from the animal's eye and had an apparent aperture of $\sim 3^{\circ}$. We used a radiometer (Sanso, model 470MD) to measure the unattenuated fluxes and to determine grey wedge positions needed for isoquantal stimuli.

For electrophysiological recordings, we fixed the animals to a stage using a mixture of beeswax and resin. We made a small triangular hole in the dorsal part of the eye using a razor blade sliver. The hole was immediately covered with a drop of saline to prevent clotting. We subsequently transferred the animal to the recording cage, placed it in the rotation centre of the perimeter device and oriented the eye's sagittal axis vertically.

A glass micro-electrode filled with $1 \mathrm{M} \mathrm{KCl}$ solution (resistance 50-70 M $\Omega$ ) was inserted into the retina through the hole. A chloridised silver wire (diameter $0.1 \mathrm{~mm}$ ), inserted into an obliquely cut antenna, functioned as the indifferent electrode. Electrical potentials were transduced with a Nihon Kohden bridge amplifier and sampled at $1 \mathrm{kHz}$ rate with a laboratory interface (BioPac Systems, model MP-150) controlled with the software package AcqKnowledge (BioPac Systems). The micro-electrode was then slowly advanced until a drop in the potential to about -40 to $-60 \mathrm{mV}$ indicated cell impalement. The visual axis of the impaled cell was determined using dim white test flashes delivered from the light guide attached to the perimeter device. We selected 73 out of about 200 recordings, where a stable resting potential, saturating responses of at least $25 \mathrm{mV}$ above the resting potential at the highest intensity and a hyperpolarising component of less than a few $\mathrm{mV}$ demonstrated that the recordings were from viable single cells. Usually, we were able to hold a cell for about 5-30 min. The hyperpolarising component in the green part of the spectrum, which was either due to an ERG artefact or due to crosstalk from neighbouring cells (Matić 1983), was most pronounced in the recordings from UV (Fig. 1) and red cells.

Three different stimulation protocols were employed: a spectral scan with roughly isoquantal stimuli, an intensity calibration and a polarisation scan. In all protocols, light pulses of $30 \mathrm{~ms}$ duration were used (Figs. 1, 2). After impaling the photoreceptor, the 23 wavelengths were scanned, from 300 to $740 \mathrm{~nm}$ and back, with light pulses spaced $1 \mathrm{~s}$ apart. The position of the grey wedge was adjusted for each stimulus, so that at all wavelengths photon fluxes were approximately equal. Usually, we recorded two or three spectral scans in the same cell. Subsequent to the first spectral scan, an intensity calibration protocol was run at a wavelength close to the main sensitivity peak of the impaled photoreceptor. In case the cell had a pronounced secondary peak, we carried out a second run at a wavelength close to that secondary peak. The intensity calibration protocol started at $4 \log$ unit attenuation and continued in steps of quarter $\log$ unit increments until the maximal intensity was reached, yielding a total of 17 intensity stimuli. Pulse intervals were shorter with low-intensity stimuli and longer with high-intensity stimuli to minimise adaptation effects (see Fig. 1). In the third polarisation scan protocol, a UV-capable polariser was inserted in front of the light guide, starting in the vertical e-vector position. The protocol consisted of 41 pulses spaced $1 \mathrm{~s}$ apart. After the first five pulses, which were ignored in the analysis, the polariser started rotating counterclockwise from the eye's aspect in $10^{\circ}$ steps and eventually finished again in the vertical e-vector position. Each protocol took $\sim 40 \mathrm{~s}$ to finish.

\section{Data analysis}

Data were imported into Matlab using the routine loadacq.m (by Mihai Moldovan, available at the Matlab Central site), and were further analysed using our own routines. The voltage responses were temporally aligned to the shutter opening time, and the baseline was calculated as the average of the 100 samples in the first $100 \mathrm{~ms}$ before the stimulus. The response amplitude was measured as the difference between the baseline and the $95 \%$ quantile of 100 samples around the peak response. The curve of response amplitudes $V(I)$ was then fitted with a Hill sigmoid $V(I)=V_{\mathrm{p}} I^{h} /\left(R^{h}+I^{h}\right)$ using the least-squares optimisation method. Here, the independent variable $I$ is the light intensity, the parameter $V_{\mathrm{p}}$ is the peak response, $h$ is Hill's slope and $R$ (or $I_{50}$ ) is the intensity where the response is half maximal (Laughlin 1981). The effective intensities $I(V)$ of tested stimuli [evoking response amplitudes in the range $\left.\left(0-V_{\mathrm{p}}\right)\right]$ were then estimated with the inverse function $I(V)=R\left[V /\left(V_{\mathrm{p}}-V\right)\right]^{1 / h}$. 

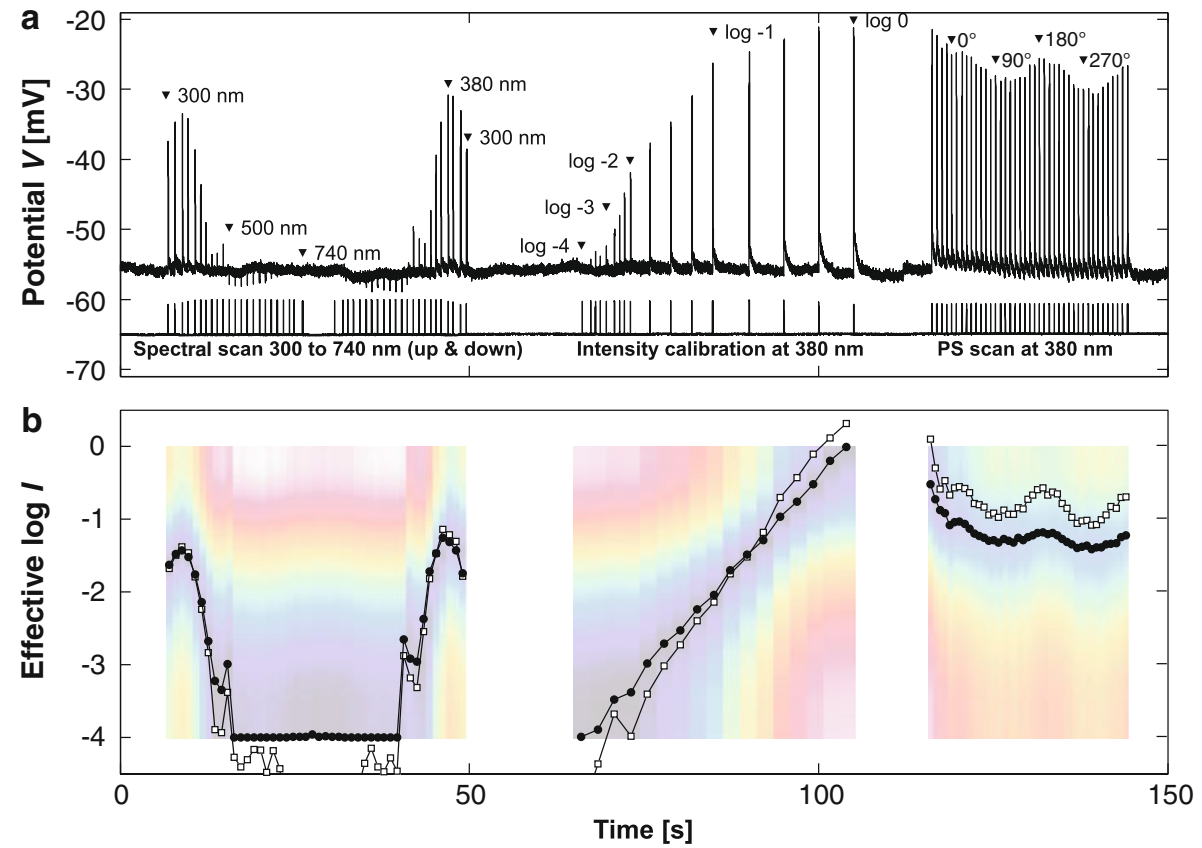

Fig. 1 Intracellular recording from a cell classified as a UV receptor. a The sequence starts with a spectral scan of 23 approximately isoquantal light pulses from 300 to $740 \mathrm{~nm}$ spaced $20 \mathrm{~nm}$ apart. This is followed with a scan going back from 740 to $300 \mathrm{~nm}$. The responses in the green wavelength region are slightly hyperpolarising, possibly due to an ERG artefact or electrical crosstalk from neighbouring green receptors. The intensity calibration scan consists of responses to light pulses at $380 \mathrm{~nm}$, covering an intensity range of $4 \log$ units, increasing in $0.25 \mathrm{log}$ unit steps. The polarisation sensitivity scan was also per-

\section{The Response Waveform Comparison method}

As an alternative for the estimation of effective intensities of light pulses (that is, the intensities of light pulses that would elicit the same response as during the calibration run), we developed the RWC method, in which the whole response to a test stimulus is compared to an array of interpolated responses from the intensity calibration protocol. The effective intensity of the tested stimulus is estimated as that of the most similar interpolated response in a least-squares sense. We created the interpolated array from the 17 measured calibration responses (Fig. 2a), by fitting a ninth degree polynomial to the instantaneous intensity-response curve $V\left(I ; t=t_{\mathrm{i}}\right)$ at each of the 100 sample points $t_{\mathrm{i}}$ where the voltage responses were significant. The calibration responses were interpolated to $0.01 \log I$ steps over the range $\log I=-4$ to $\log I=0$, thus yielding an array with a size of $401 \times 100$ points (Fig. 2 b, c). The tested response was compared to each of the interpolated responses (Fig. 2d, e). The effective intensity of the test response was estimated to be one of the most similar responses from the array, that is, of the interpolated response having the minimal root mean square sample-per-sample difference formed at $380 \mathrm{~nm}$. It starts with five adaptation prepulses, which are followed by 36 pulses with the polariser being rotated in $10^{\circ}$ steps. The duration of each of the three protocols was $\sim 40 \mathrm{~s}$. b Estimation of effective intensity of the light pulses. The background waterfall plot shows the root mean square difference (RMSD) profiles (see Fig. 2e, f). The RMSD minima are estimates of effective intensities obtained by the RWC method (black circles). Open squares show the estimates obtained by reverse transformation of a Hill sigmoid

with the tested response (RMSD; Fig. 2e, f). We note that in the given example (Fig. 2d, e, f), we perform selfvalidation of the method by estimating the effective intensities of the responses that were used in the calibration run proper.

The accuracies of the RWC and the conventional Hill-fit method were compared by rerunning the calibration data as the tested data (Fig. 2e, f) and performing linear regression between the applied and estimated pulse intensity. We concluded that the RWC method outperforms the conventional method with reverse transformation, especially in the lowand high-intensity range (Figs. 1b, 3), and we therefore used the RWC method in the calculations of the spectral and polarisation sensitivities.

Fitting of spectral and polarisation sensitivity profiles

In the case of spectral scans, the estimated effective intensity of the tested response was corrected for any remaining flux differences. The estimated effective intensities were anti-log transformed, the values from the forward and the backward scan were averaged, and the spectra from each cell were normalised to unity area to give equal weights to all measured cells. 

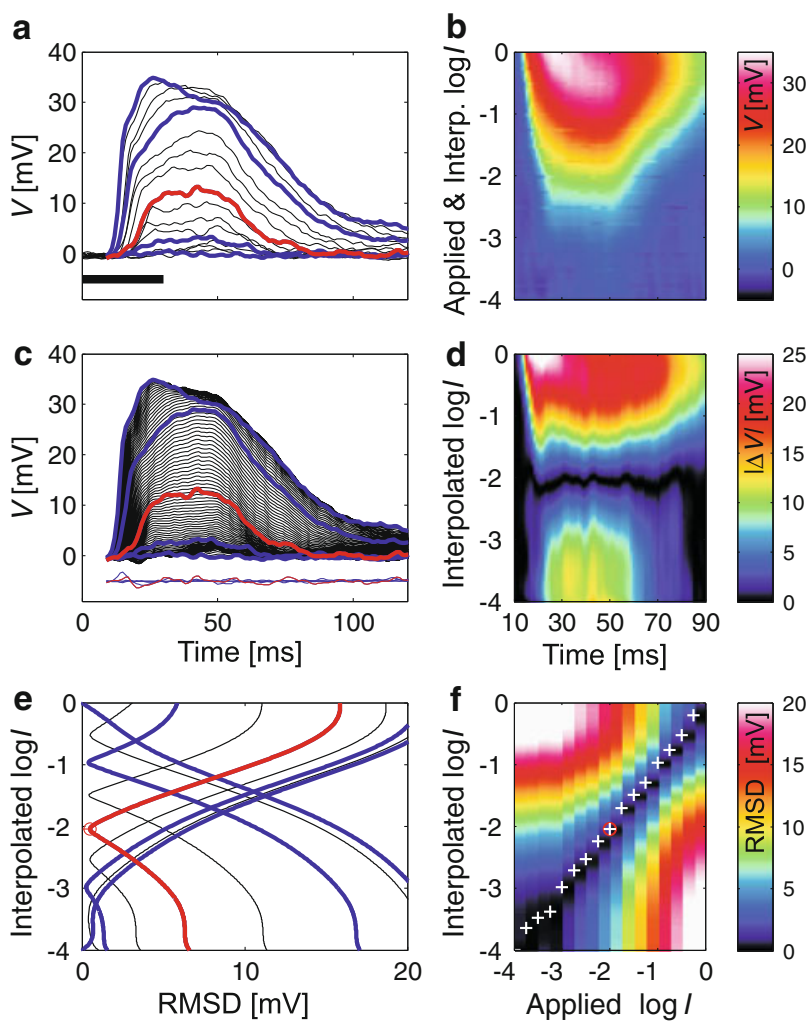

Fig. 2 The response waveform comparison (RWC) method. a, b, c The interpolation of the calibration responses. $\mathbf{d}, \mathbf{e}, \mathbf{f}$ The principle of estimation of effective intensities of the responses. As an example, we estimated the effective intensities of the responses from the same calibration run to perform self-validation of the method. a Measured calibration responses to light pulses with intensity increasing in $0.25 \mathrm{log}$ unit steps over a range of $4 \log$ units (from -4 to 0 ). The response at $\log I=-2$ is shown in red, and the responses at $\log I=-4,-3,-1$ and 0 are given in blue. The black line marks the shutter opening. b Waterfall plot of the interpolated response array. The measured responses are shown interlaced at their nominal intensities. $\mathbf{c}$ Comparison between the measured and interpolated responses. Interpolated responses (spaced $0.05 \log$ unit apart) are shown in black. The measured responses are shown as in $\mathbf{a}$. The inset below the responses shows the difference between the measured and interpolated responses. d Waterfall plot showing the absolute sample-wise difference between the measured response to a light pulse at intensity $\log I=-2$ (red trace in c) and the interpolated response array (shown in b). As expected, the sample-wise difference is close to zero (black colour) in the region around $\log I=-2$. See also the red RMSD profile in e. e RMSD profiles (root mean square sample-wise difference between the measured response and each of the interpolated responses) for some of the calibration stimuli. The positions of the minima of the RMSD profiles were used as the estimators of the effective intensities of the applied stimuli. The red curve presents the RMSD profile for the response to a light pulse at $\log I=-2$ (compare with d). The RMSD minimum for the measured response $(0.47 \mathrm{mV}$ ) is reached at $\log I=-2.04$ (red circle in e, f). Blue curves, showing the RMSD profiles for the measured responses at $\log I=-4,-3,-1$ and 0 , and the black thin curves (responses to intensities at $0.5 \log$ units in between) have their minima close to the applied intensities. f Waterfall plot of the RMSD profiles of all the measured responses from the calibration run. The white crosses, representing the minima of RMSD curves for each stimulus (repeated as solid blue diamonds in Fig. 3), show a close correlation between the applied and estimated intensity (colour figure online)

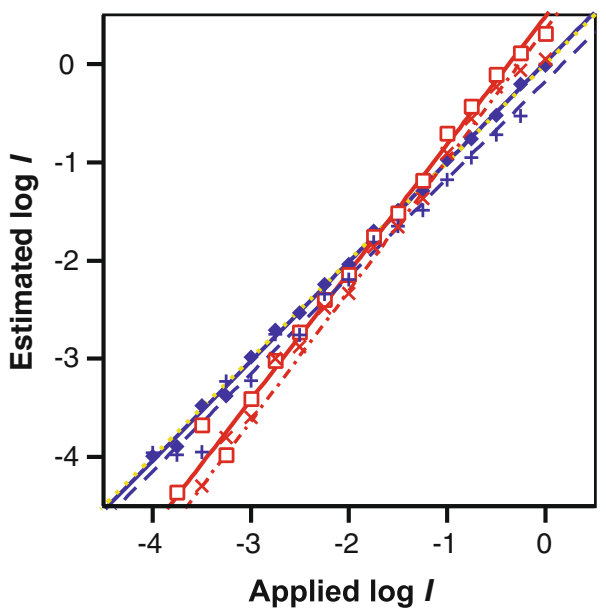

Fig. 3 Self-validation comparison of the RWC and Hill method. Comparison of the accuracy of the effective stimulus estimation based on retesting the calibration stimuli. The RWC estimates (blue circles, linear regression $y=1.014 x+0.018$; thick blue line) are closer to the ideal 1:1 relation between the applied and estimated intensity than the estimates obtained via the inverse Hill transformation (red squares, $y=1.302 x+0.484$; thick red line). The Hill sigmoid fit to the intensityresponse curve (not shown) was tight, with $V_{\mathrm{p}}=37.0 \pm 0.6 \mathrm{mV}$, $\log R=-1.65 \pm 0.03$ and $h=0.75 \pm 0.03$ (mean $\pm \mathrm{SE}$ ). The red and blue crosses show the estimates obtained from a second intensity-response series (not shown in Fig. 1), compared with the calibration stimuli. The regression lines for these two series are shown with thin blue and red lines. Again, the RWC estimates are closer to 1:1 relation than those obtained from inverse Hill transformation (colour figure online)

Spectral sensitivities of green, violet, narrow blue and UV receptor classes were fitted with a rhodopsin template (Govardovskii et al. 2000), which requires a single parameter (the peak wavelength, $\lambda_{\mathrm{p}}$ ). When the spectral sensitivity curve deviated from the rhodopsin template in the shortwavelength range, we fitted only the long-wavelength part of the sensitivity spectrum, because the slope of the long-wavelength tail of the rhodopsin absorption spectra is not very sensitive to optical filtering effects, but critically depends on the rhodopsin peak wavelength (Wakakuwa et al. 2004; Arikawa et al. 2005). Additionally, since the peak wavelengths and bandwidths of the receptor classes may well be different from those obtained from template fitting, we also fitted the spectral sensitivity curves with a heuristic template, based on the asymmetric Gaussian function $S(\lambda)=a_{\mathrm{p}} \exp \left\{[-4 \ln (2)]\left[\left(\lambda-\lambda_{\mathrm{p}}\right) / \delta\right]^{2}\left[1+\varepsilon\left(\lambda-\lambda_{\mathrm{p}}\right) / \delta\right]^{-2}\right\}$, where $a_{\mathrm{p}}$ is the peak amplitude, $\delta$ the bandwidth (FWHM), and $\varepsilon$ the asymmetry factor; the function was modified from Peak Fitter software (by Marcus Karolewski). We used a single asymmetric Gaussian function for single-peaked cell classes (UV, violet). A sum of two Gaussian functions was used for fitting double-peaked receptor classes (green, red). The parameter indices 1 and 2 in Table 2 designate the primary and the secondary peak, respectively. The fitting was done 
either with linear or logarithmically transformed sensitivity data. For the red classes, we further derived two parameters: the relative amplitude of the secondary peak, $a_{2} / a_{1}$ and the left cutoff wavelength (wavelength at the left half maximum, WLHM) of the main peak, $\lambda_{1}-\delta_{1} / 2$. For the linear fit of double-peaked receptors, the secondary peak wavelength was fixed to $\lambda_{2}=363 \mathrm{~nm}$ and its bandwidth to $\delta_{2}=110 \mathrm{~nm}$.

The effective sensitivities measured in the polarisation scan were fitted with the function $S(\theta)=a_{\mathrm{m}} K\left[1+\psi\left(2 \cos ^{2}\right.\right.$ $(\zeta-\theta)-1)]$, where $\theta$ is the angular orientation of the polariser. The parameters are $\zeta$, the polarisation sensitivity (PS) axis angle (the angle of maximal sensitivity to linearly polarised light, range between $0^{\circ}$ and $180^{\circ}$ ), and $\psi$, the relative polarisation sensitivity amplitude (a dimensionless quantity between 0 and 1 , describing the modulation around the mean response $a_{\mathrm{m}}$ ). The polarisation sensitivity ratio $P_{\mathrm{s}}$ is calculated from $\psi$ as $P_{\mathrm{s}}=(1+\psi) /(1-\psi)$. The correction factor, $K=1+k N$ describes a linear adaptation trend, depending on the pulse count, $N$. Parameter indices 1 and 2 (Table 2) designate the parameter estimates for the primary and secondary peak, respectively. We note that it has been shown that the orientation of microvilli determines the PS axis angle, $\zeta$, of the photoreceptor (Bandai et al. 1992).

The peak wavelength estimators obtained from template and heuristic fitting to the spectra obtained from all the cells in a receptor class are reported with $95 \%$ confidence intervals. The estimators for polarisation sensitivity for single cells are reported with their standard errors (SE). Group averages of these estimators are reported with standard deviations (SD) without error propagation.

\section{Results}

Spectral sensitivity classes

We analysed 73 cells and classified their spectral sensitivities into nine classes (Fig. 4), based on the shape of the spectral sensitivity curve and its peak wavelength, $\lambda_{\mathrm{s}}$. The spectral sensitivities of four classes resembled the template spectrum of rhodopsin (Govardovskii et al. 2000), namely the ultraviolet (UV, $\lambda_{\mathrm{s}} \approx 360 \mathrm{~nm}$ ), violet $\left(\mathrm{V} ; \lambda_{\mathrm{s}} \approx 420 \mathrm{~nm}\right)$, broad green and narrow green (bG and $\mathrm{nG} ; \lambda_{\mathrm{s}} \approx 560 \mathrm{~nm}$ ) receptors. The shape of the sensitivity spectra of the remaining photoreceptor classes distinctly deviated from the rhodopsin template. The following classes were single peaked: the narrow blue $\left(\mathrm{nB} ; \lambda_{\mathrm{s}} \approx 460 \mathrm{~nm}\right)$, the blunt broad blue $\left(\mathrm{bBb} ; \lambda_{\mathrm{s}} \approx 500 \mathrm{~nm}\right)$ and the sharp broad blue (bBs; $\left.\lambda_{\mathrm{s}} \approx 500 \mathrm{~nm}\right)$. The red receptors $\left(\lambda_{\mathrm{s}} \approx 640 \sim 680 \mathrm{~nm}\right)$ had a clear secondary peak of $10-50 \%$ at $\sim 480 \mathrm{~nm}$. The peak wavelength of the red receptors varied considerably suggesting that this class contained more than one receptor

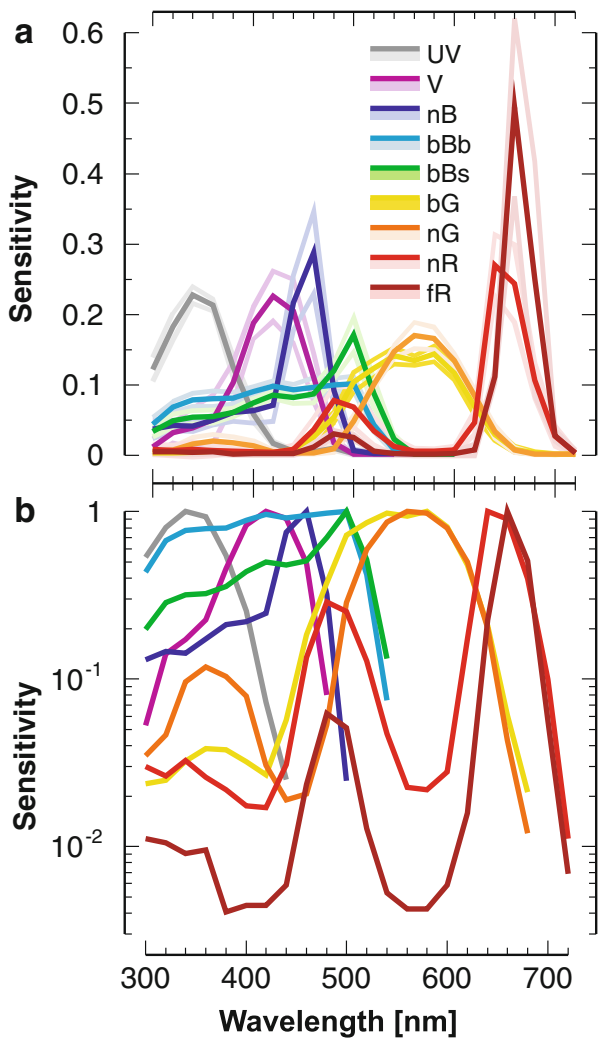

Fig. 4 Spectral sensitivities. a Spectral sensitivities of the set of the nine photoreceptor classes of $C$. erate normalised to their area. The area between the faint lines represents standard deviations. b Spectral sensitivities normalised to the peak, with a logarithmic ordinate. Error bars are omitted here for the sake of clarity. Number of measured cells $(n)$ and individuals $(m)$ for all classes is given as $(n, m)$ : UV $(13,9)$; V $(4,4)$; nB $(6,6)$; bBb $(6,5)$; bBs $(6,3)$; nG $(9,9)$; bG $(21,10)$; nR $(6$, 5), fR $(2,2)$

type, which was also supported by the anatomy (Arikawa et al. 2009). Thus, we have divided the red receptor class into a near red (nR) and a far red (fR) class.

Spectral sensitivities are commonly presented normalised at their peak value, but Fig. 4a presents the sensitivities normalised to their area, so that more spectrally selective receptor classes with narrow bandwidths have higher peaks. The most spectrally selective receptor classes were $\mathrm{nB}, \mathrm{nR}$ and $\mathrm{fR}$, with FWHM (full-width at half maximum) of the main peak at $\sim 50 \mathrm{~nm}$. The most broadband receptor is $\mathrm{bB}$, covering a wide wavelength range (FWHM $\approx 140 \mathrm{~nm}$, from 300 to $540 \mathrm{~nm}$ ). A semi-logarithmic representation of the sensitivity spectra (Fig. 4b) reveals for the nG class a deep trough in the $440-460 \mathrm{~nm}$ range, whilst the bG class has an extremely low UV sensitivity.

In the next step, we estimated the peak absorption wavelengths of the expressed rhodopsins. The sensitivity spectra of the UV, V, bG and nG classes resemble rhodopsin templates, but there are considerable deviations, especially in the UV range. We therefore limited the template fit to the 
Table 1 Rhodopsin template fits

\begin{tabular}{llllll}
\hline Class & Linear fit & Log fit & $n$ & Fit range & $\begin{array}{l}\lambda_{\operatorname{lin}}-\lambda_{\log } \\
(\mathrm{nm})\end{array}$ \\
\hline & $\lambda_{\operatorname{lin}}(\mathrm{nm})$ & $\lambda_{\log }(\mathrm{nm})$ & & & \\
\hline $\mathrm{UV}$ & $355 \pm 1.6$ & $362 \pm 1.3$ & 13 & $340-400$ & -7 \\
$\mathrm{~V}$ & $422 \pm 3.9$ & $413 \pm 3.3$ & 4 & $400-500$ & 9 \\
$\mathrm{nB}$ & $421 \pm 7.8$ & $413 \pm 6.7$ & 5 & $460-500$ & 8 \\
$\mathrm{bBb}$ & $461 \pm 4.9$ & $457 \pm 4.1$ & 6 & $500-560$ & 4 \\
$\mathrm{bBs}$ & $467 \pm 6.0$ & $453 \pm 6.4$ & 6 & $500-560$ & 14 \\
$\mathrm{nG}$ & $568 \pm 1.7$ & $558 \pm 1.9$ & 9 & $520-680$ & 10 \\
$\mathrm{bG}$ & $562 \pm 1.3$ & $560 \pm 1.3$ & 20 & $520-680$ & 2 \\
$\mathrm{nG}+\mathrm{bG}$ & $564 \pm 1.1$ & $559 \pm 1.1$ & 29 & $520-680$ & 5 \\
\hline
\end{tabular}

The ranges of estimators are given as $95 \%$ confidence intervals

peak and the long-wavelength limb range. In addition to fitting the sensitivity spectra in the linear domain, we fitted the log-transformed spectra as well, thus giving a higher weight to the values in the long-wavelength limb of the spectra. We note that when fitting in the logarithmic domain, the rhodopsin estimates were biased towards shorter wavelengths in all but the UV class (Table 1; ranges are given for symmetric $95 \%$ confidence intervals).

Fitting the sensitivity spectra of the UV receptor class yielded $355 \mathrm{~nm}$ (logarithmic fit: $362 \mathrm{~nm}$ ) for the peak wavelength of the UV rhodopsin (Fig. 5a). Similarly, fitting the spectra of the $\mathrm{V}$ receptor class yielded a peak wavelength of a violet/blue rhodopsin of $422 \mathrm{~nm}$ (logarithmic fit: $413 \mathrm{~nm}$; Table 1). A very similar value was obtained by fitting the right limb of the $\mathrm{nB}$ spectra (Fig. 5c). This suggests that at least one of the $\mathrm{CeV} 1$ and $\mathrm{CeV} 2$ rhodopsins peaks in the violet range, around $420 \mathrm{~nm}$.

Although the sensitivity spectra of the $\mathrm{bBb}$ and $\mathrm{bBs}$ receptor classes strongly deviated from predictions by the rhodopsin template, the long-wavelength limbs of the spectra, when fitted with the rhodopsin template, gave similar estimates for the underlying rhodopsin: 461 and $467 \mathrm{~nm}$, respectively, for the linear fit; 457 and $453 \mathrm{~nm}$, respectively, for the logarithmic fit

b
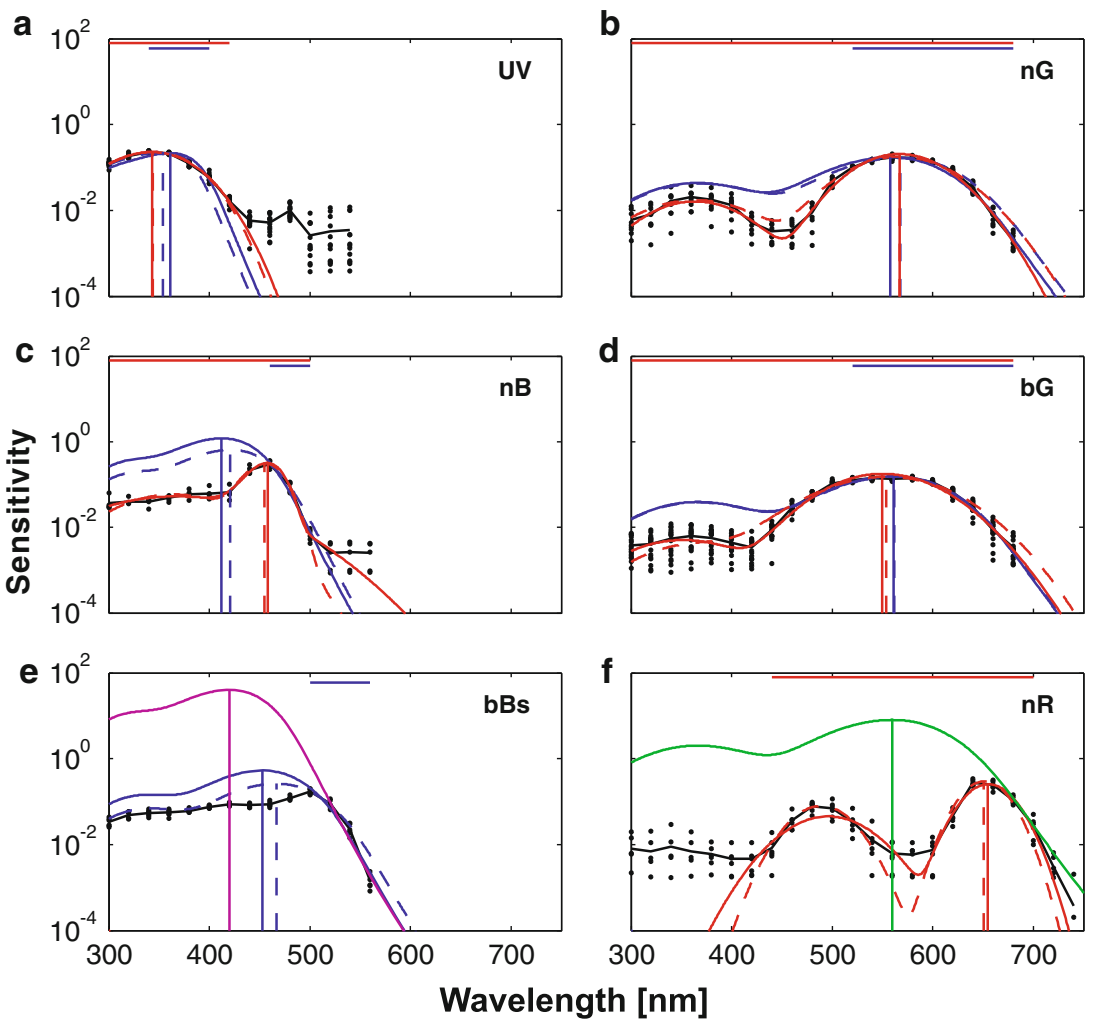

Fig. 5 Fits to the sensitivity profiles of $U V, n B, b B s, n G, b G$ and $n R$ classes. The measured log sensitivity spectra (black dots; black line shows the average) were fitted with the rhodopsin templates (blue lines) and heuristic asymmetric Gaussian functions (red lines). The fitting was performed both in the logarithmic (solid lines) and in the linear domain (dashed lines). The vertical lines designate the peak wavelengths obtained with different fits. The horizontal bars designate the interval of data used for fitting the template spectra (blue lines) and heuristic fits (red lines). The $\mathrm{UV}, \mathrm{nB}$ and bBs sensitivity spectra in the left-hand column (a, c, e) yielded predictions for rhodopsins peaking in the UV, violet and blue, respectively (see Table 1). The magenta line in e, showing the template of a rhodopsin peaking at $420 \mathrm{~nm}$, was fitted by eye. In the right-hand column, the $\mathrm{nG}$ and the bG spectra (b, d) yielded predictions for a rhodopsin absorbing maximally at $560 \mathrm{~nm}$ (see Table 1). The green line in (f), showing this rhodopsin spectrum, was fitted to the sensitivity profile of the $\mathrm{nR}$ class by eye. The numerical fitting results for all classes are shown in Tables 1, 2, and 3. Fits to classes $\mathrm{V}$ and $\mathrm{bBb}$ are not shown. Individual fits to $\mathrm{nR}$ and $\mathrm{fR}$ classes are shown in Fig. 10 (colour figure online) 
(Fig. 5e). This suggests that one of the $\mathrm{CeV} 1$ or $\mathrm{CeV} 2$ rhodopsins peaks in the blue, at around $460 \mathrm{~nm}$.

The estimates for the rhodopsin peak wavelength of the $\mathrm{nG}$ and bG classes were similar (Fig. 5b, d), in line with the finding that $C$. erate expresses only a single green rhodopsin, CeL (Awata et al. 2009). The joint estimate for the peak wavelength of CeL was assessed to be around $560 \mathrm{~nm}$ (linear estimate $564 \mathrm{~nm}$; logarithmic estimate $559 \mathrm{~nm}$ ).

Heuristic fitting of the spectral sensitivities

The fitting procedure with a rhodopsin template may have well predicted the underlying rhodopsins, but the sensitivity profiles are not described accurately by the template spectra. We therefore decided to find heuristic fits that would accommodate the measured spectra in the whole wavelength range. Figure 5 shows heuristic fits in the linear (dashed red curves) and logarithmic domains (solid red curves). Table 2 summarises the results of the heuristic fitting in the logarithmic domain for $\mathrm{UV}, \mathrm{V}, \mathrm{nB}, \mathrm{nG}$ and $\mathrm{bG}$ receptor classes; the estimators obtained by fitting in the linear domain were essentially the same.

An asymmetric single Gaussian function (see "Methods") fitted to the sensitivity spectra of the UV class (Fig. 5a) yielded a peak wavelength at around $345 \mathrm{~nm}$, somewhat shorter than the estimate from the rhodopsin template fit. An asymmetric Gaussian function fitted to the sensitivity spectra of the $\mathrm{V}$ class yielded a peak wavelength at around $425 \mathrm{~nm}$, both in the logarithmic domain (Table 2) and in the linear domain (not shown), close to the result of the linear rhodopsin template fit.

For heuristic fitting of the sensitivity spectra of the $n B$, $\mathrm{bG}$ and $\mathrm{nG}$ classes, we used a sum of an asymmetric and a symmetric Gaussian function to fit the main and the secondary peaks, respectively (see "Methods"). In most cases, the asymmetricity of the main peak was negligible. The main peak wavelength for the $\mathrm{nB}$ class was estimated at $\sim 456 \mathrm{~nm}$, with a very narrow bandwidth (FWHM, $\delta_{1}$ ) of $39 \mathrm{~nm}$ (Fig. 5c). We were unable to find a sensible heuristic fit for the sensitivity profiles of $\mathrm{bBb}$ and $\mathrm{bBs}$ classes.
The main peak wavelength $\left(\lambda_{1}\right)$ of the $\mathrm{nG}$ class was $\sim 567 \mathrm{~nm}$ (Fig. 5b), somewhat longer than the 559 and $563 \mathrm{~nm}$ of the template fits. The relative $\beta$-peak sensitivity $(0.08)$ was substantially lower than that predicted by the rhodopsin template (0.25). Because of a reduced sensitivity in the blue range (Fig. 4b), the bandwidth (FWHM) of the main peak was narrower than that of the template for Rh560 (Table 2).

The main peak wavelength $\left(\lambda_{2}\right)$ of the bG class was at $\sim 550 \mathrm{~nm}$, somewhat shorter than the template fit values, whilst the bandwidth (FWHM) was close to the one predicted by the template. In this class, the relative sensitivity of the second peak $\left(a_{2} / a_{1}\right)$ was $\sim 0.02$ (Fig. $5 \mathrm{~d}$ ), lower than that of the $\mathrm{nG}$ class (Fig. 5b). We noticed that the distribution of $a_{2} / a_{1}$ in this class was bimodal, with about half of the cells (bG1) having $a_{2} / a_{1} \approx 0.05$ and the other half (bG2) virtually at the detection threshold of the RWC method (Table 2). In our further analyses, we however have considered these two subclasses together.

\section{Red cells}

We fitted the spectral sensitivity profiles of red cells with a double Gaussian function. We divided the eight measured red cells into the near red class (nR, 6 cells, Figs. 5f, 10; Table 3$)$, with the main peak $\left(\lambda_{1}\right)$ at $\sim 651 \mathrm{~nm}$ and cutoff $\left(\lambda_{1}-\delta_{1} / 2\right)$ at $\sim 629 \mathrm{~nm}$, and the far red class (fR, 2 cells, Fig. 10; Table 3), with the main peak at $664 \mathrm{~nm}$ and cutoff at $648 \mathrm{~nm}$. All nR and fR cells had a secondary peak $\left(\lambda_{1}\right)$ at $\sim 480 \mathrm{~nm}$. In "Discussion", we substantiate the division into two classes, despite low cell numbers, by further analysing the sensitivity profiles of individual cells and relating their main peak and cutoff wavelengths to the relative amplitudes of the secondary peaks $\left(a_{2} / a_{1}\right)$.

\section{Polarisation sensitivity}

We have determined the relative polarisation sensitivity amplitude (PS amplitude, $\psi$ ) and the angle of maximal polarisation sensitivity (PS axis angle, $\zeta$ ) for all receptor classes (Fig. 6). For the UV, V and bG classes, we obtained

Table 2 Heuristic fits of UV, blue and green classes

\begin{tabular}{llllllllll}
\hline Class $\log$ fit & $\lambda_{1, \log }(\mathrm{nm})$ & $\delta_{1}(\mathrm{~nm})$ & $\varepsilon_{1}$ & $a_{2} / a_{1}$ & $\lambda_{2}(\mathrm{~nm})$ & $\delta_{2}(\mathrm{~nm})$ & $n$ & Fit range & $\lambda_{1, \text { lin }}-\lambda_{1, \log }(\mathrm{nm})$ \\
\hline $\mathrm{UV}$ & $343 \pm 2$ & $85 \pm 5$ & $0.08 \pm 0.07$ & & & & 13 & $300-440$ & 1 \\
$\mathrm{~V}$ & $426 \pm 8$ & $78 \pm 6$ & $0.33 \pm 0.10$ & & & 4 & $300-500$ & 0 \\
$\mathrm{nB}$ & $458 \pm 5$ & $36 \pm 6$ & $0.19 \pm 0.24$ & $0.18 \pm 0.05$ & $366 \pm 10$ & $152 \pm 22$ & 5 & $300-500$ & -2 \\
$\mathrm{nG}$ & $567 \pm 5$ & $85 \pm 3$ & $-0.02 \pm 0.05$ & $0.08 \pm 0.02$ & $364 \pm 4$ & $92 \pm 9$ & 9 & $300-680$ & 0 \\
bG1 & $552 \pm 3$ & $111 \pm 3$ & $0.00 \pm 0.04$ & $0.05 \pm 0.01$ & $351 \pm 4$ & $95 \pm 12$ & 10 & $300-680$ & 2 \\
bG2 & $550 \pm 4$ & $96 \pm 3$ & $-0.03 \pm 0.04$ & $0.01 \pm 0.00$ & $349 \pm 13$ & $148 \pm 62$ & 10 & $300-680$ & 5 \\
Rh560 & 558 & 112 & 0.12 & 0.25 & & & & \\
\hline
\end{tabular}

The ranges of estimators are given as $95 \%$ confidence intervals. The last line shows a fit to a rhodopsin template peaking at $560 \mathrm{~nm}$ 
Table 3 Heuristic fits for the red receptors in the linear domain

\begin{tabular}{lllllll}
\hline Class & $\lambda_{1}(\mathrm{~nm})$ & $\delta_{1}(\mathrm{~nm})$ & $a_{2} / a_{1}$ & $\lambda_{2}(\mathrm{~nm})$ & $\delta_{2}(\mathrm{~nm})$ & $\lambda_{1}-\delta_{1} / 2(\mathrm{~nm})$ \\
\hline $\mathrm{nR}$ & $651 \pm 1$ & $44 \pm 3$ & $0.27 \pm 0.06$ & $489 \pm 6$ & $57 \pm 14$ & $629 \pm 3$ \\
$\mathrm{fR}$ & $664 \pm 2$ & $32 \pm 5$ & $0.06 \pm 0.11$ & $487 \pm 38$ & $45 \pm 90$ & $648 \pm 5$ \\
\hline
\end{tabular}

The ranges of estimators are given as $95 \%$ confidence intervals

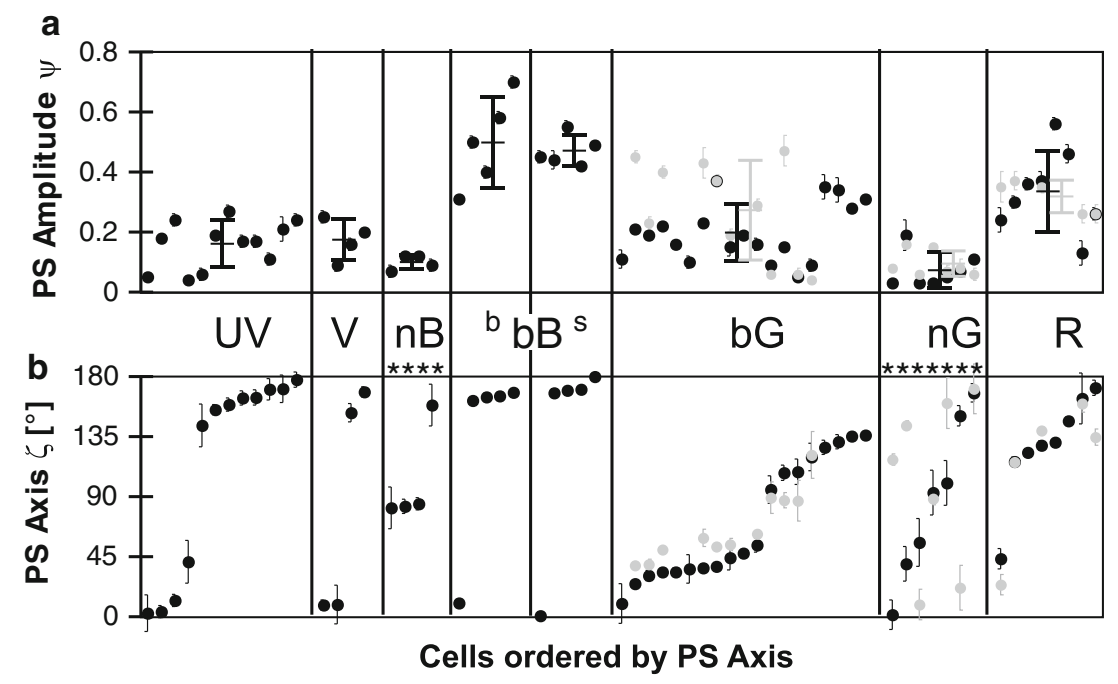

Fig. 6 Analysis of polarisation sensitivities (PS). a Relative polarisation sensitivity amplitudes $(\psi)$. b Angles of maximal polarisation sensitivity (PS axis angles, $\zeta$ ). The panels are sorted by the receptor class and $\zeta$. Black points represent the estimators $\zeta_{1}$ and $\psi_{1}$ measured close to the primary sensitivity peak $(380,420,460,500,560$ and $640 \mathrm{~nm})$. Grey points represent the estimators at the secondary sensitivity peak,

$\psi=0.15 \sim 0.20$ (Fig. 6a), and thus a polarisation sensitivity ratio of $P_{\mathrm{s}} \approx 1.3-1.5$ (see "Methods"). The most polarisation-sensitive receptor classes were $\mathrm{bBb}, \mathrm{bBs}$ and $\mathrm{R}$ : $\psi \approx 0.30-0.50$, so that $P_{\mathrm{s}} \approx 1.8-3.0$. The polarisation axes of four classes ( $\mathrm{UV}, \mathrm{V}, \mathrm{bBb}, \mathrm{bBs}$ ) appear to be aligned around the vertical axis, $\zeta \approx 0^{\circ}$ or $180^{\circ}$ (Fig. 6b). The PS axis angles of the $\mathrm{R}$ and $\mathrm{bG}$ receptor classes appear to be oriented at diagonal positions, $\zeta=135^{\circ}$ and $\zeta=45^{\circ}$, both at the primary and the secondary sensitivity peaks (Fig. $6 \mathrm{~b}$, black and grey points, respectively). The $\mathrm{nG}$ and $\mathrm{nB}$ receptor classes had very low polarisation sensitivities $(\psi \approx 0.10$, or $P_{\mathrm{s}} \approx 1.2$; Fig. 6a). Consequently, the estimates of the polarisation axis $\zeta$ in these classes mostly have large standard errors and we consider them to be unreliable (asterisks in Fig. 6b); we show them for the sake of completeness.

In some cells of the $\mathrm{bG}$ and $\mathrm{R}$ classes, we were able to measure the polarisation sensitivity at the primary as well as at the secondary spectral peak (black and grey points in Fig. 6, respectively). For the bG class, the polarisation sensitivities for the primary and secondary peak were measured at 560 and $380 \mathrm{~nm}$, respectively, and for the $\mathrm{nR}$ and fR cells at 660 and $480 \mathrm{~nm}$, respectively. In both classes, the relative polarisation sensitivity amplitude at the primary peak $\left(\psi_{1}\right)$ was slightly lower than the one at the secondary $\zeta_{2}$ and $\psi_{2}$ (380 and $480 \mathrm{~nm}$ for the green and red receptor classes, respectively). Thin bars indicate the standard errors of the estimators. Asterisks mark the $\mathrm{nB}$ and $\mathrm{nG}$ groups, where the polarisation axis estimates are unreliable. Thick bars in a show group averages $( \pm \mathrm{SD}) \zeta$ for the receptor classes

peak $\left(\psi_{2}\right.$; Fig. 7a), whilst the PS axis angles for the primary peak $\left(\zeta_{1}\right)$ and the secondary peak $\left(\zeta_{2}\right)$ were very similar (Fig. 7b).

Intensity-response characteristics

We analysed the intensity calibration series of 50 cells where response saturation was reached and the estimated parameters converged with acceptable errors. We fitted a Hill sigmoid to the response series. The two intensity-independent parameters of the Hill sigmoid, peak amplitude $V_{\mathrm{p}}$ and slope $h$, did not systematically depend on the receptor class (Fig. 8). For individual intensity-response curves, $V_{\mathrm{p}}$ was in the range of $26-63 \mathrm{mV}(n=50)$. The grand average over all cells for the peak amplitude was $V_{\mathrm{p}}=41 \mathrm{mV}$ $(\mathrm{SE}=0.95 \mathrm{mV})$ and for the Hill slope $h=0.73$ ( $\mathrm{SE}=0.01$; ranging from 0.55 to 0.92 for individual cells). There was a weak negative correlation $\left(r^{2}=0.24\right)$ between $V_{\mathrm{p}}$ and $h$, relating a higher peak amplitude with a shallower slope of the intensity-response function.

For all analysed cells, the intensity needed for a halfmaximal response, $R$, ranged between $\log R=-2.55$ and -0.50 (not shown). Within one receptor class (bG, $n=18$ ), the $\log R$ range was quite large, over $1.30 \log$ units (not 

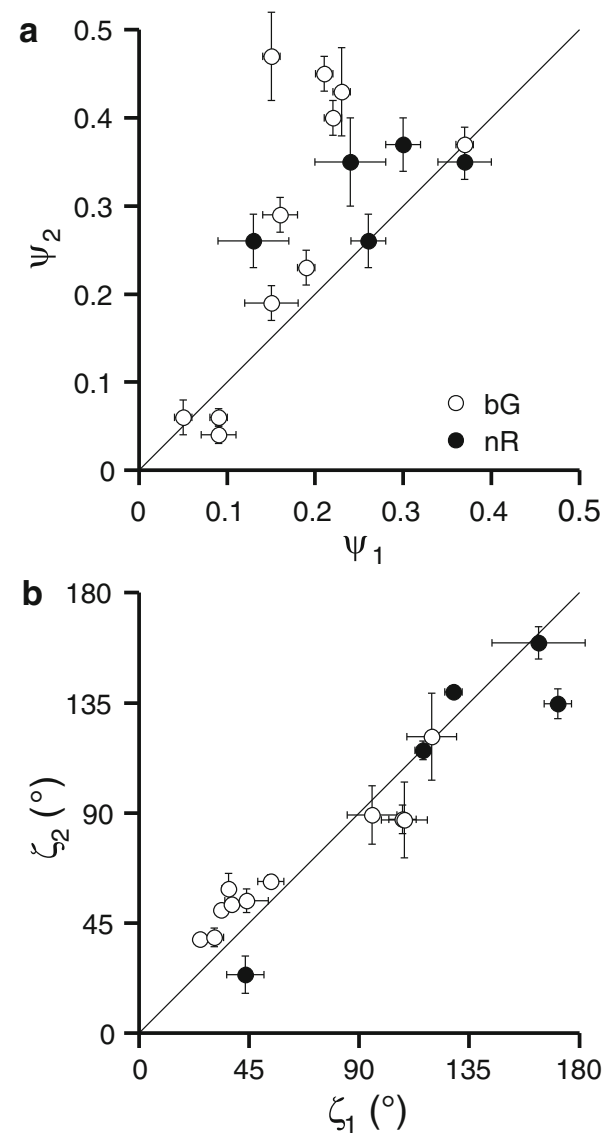

Fig. 7 Correspondence of PS amplitude and axis of bG and nR cells. a The relative polarisation sensitivity amplitudes of primary $\left(\psi_{1}\right)$ and secondary peaks $\left(\psi_{2}\right)$ of some bG (open circles) and $\mathrm{nR}$ cells (solid circles). $\mathbf{b}$ The respective PS axis angles (angles of maximal polarisation

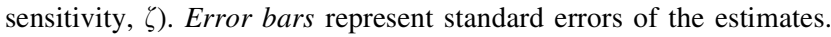
Angles $\zeta$ show good correspondence, whilst $\psi_{2}$ is on average bigger than $\psi_{1}$

shown), despite optimally adjusting the azimuth and elevation of the optical fibre output throughout all experiments.

\section{Discussion}

This study describes the set of photoreceptor classes in the eye of the Eastern Pale Clouded Yellow, Colias erate. We have classified the cells into nine receptor classes: ultraviolet (UV), violet $(\mathrm{V})$, narrow blue $(\mathrm{nB})$ and two broad blue subclasses covering the spectrum from the UV to bluegreen, one of them having a blunt sensitivity profile (bBb) and another having an additional sharp peak at $\sim 500 \mathrm{~nm}$ (bBs), two green classes ( $\mathrm{nG}$ and $\mathrm{bG}$ ) and two red classes (near red, nR; and far red, fR).

We were able to fit the long-wavelength limbs of six photoreceptor classes ( $\mathrm{UV}, \mathrm{V}, \mathrm{bG} / \mathrm{nG}$ and $\mathrm{bBb} / \mathrm{bBs}$ ) with the Govardovskii rhodopsin template (Govardovskii et al. 2000). We have obtained estimates for one UV rhodopsin

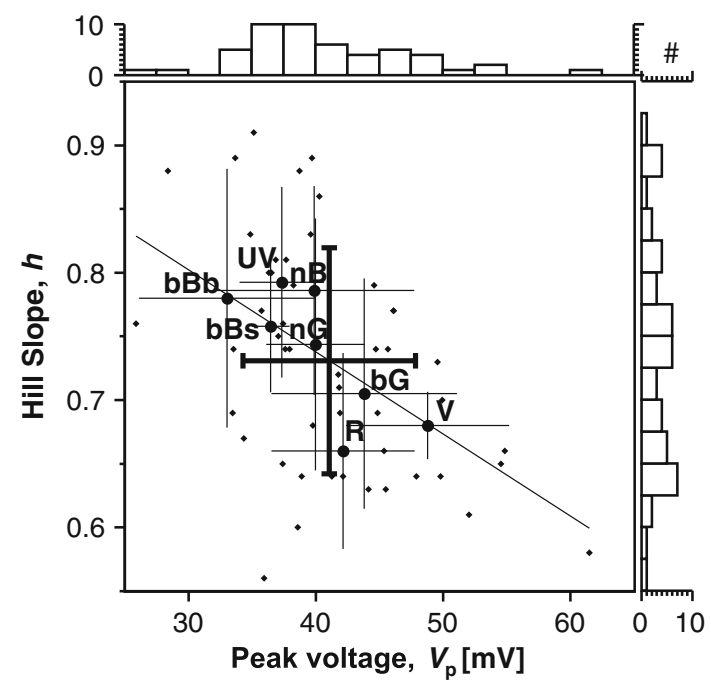

Fig. 8 Parameters of the intensity-response curve. A scatter plot of the two parameters of the Hill function, $V_{\mathrm{p}}$ (abscissa), and the slope, $h$ (ordinate). Black dots represent estimates for 50 individual cells where the function fit converged well. Solid circles with crosses are group averages (mean $\pm \mathrm{SD}$; number of cells in classes: UV 4, V 3, nB 5, $\mathrm{bBb} 3$, bBs 5, bG 18, nG 8, R 4). The thick solid cross is the grand average calculated from individual cells (mean $\pm \mathrm{SD}$ ): $V_{\mathrm{p}}=41.1 \pm$ $6.8 \mathrm{mV}$ and $h=0.73 \pm 0.09$. The regression line, based on individual cells $\left(h=0.99-0.0064 V_{\mathrm{p}} ; r^{2}=0.24\right)$, shows a weak correlation between a lower maximal response and a steeper slope. The edge histograms show the distributions of the two parameters

$\left(\lambda_{\mathrm{p}} \approx 360 \mathrm{~nm}\right)$, two violet/blue rhodopsins $\left(\lambda_{\mathrm{p}} \approx 420\right.$ and $\left.\lambda_{\mathrm{p}} \approx 460 \mathrm{~nm}\right)$ and one green rhodopsin $\left(\lambda_{\mathrm{p}} \approx 560 \mathrm{~nm}\right)$, which presumably correspond to the four rhodopsins found by mRNA hybridisation (CeUV, CeV1, CeV2 and CeL; Awata et al. 2009). Using these data and the eye anatomy data (Arikawa et al. 2009), we discuss our results. We compare the so far assembled data on $C$. erate with the more extensive knowledge of Pieris rapae.

The UV receptor class

The PS axis angle of the UV receptor class was found to be around the vertical axis, $\zeta \approx 0^{\circ}\left(180^{\circ}\right)$. The peak wavelength of the CeUV rhodopsin following from the Govardovskii template fits to the sensitivity profile, $\sim 360 \mathrm{~nm}$, and coincides with the peak wavelength of the PrUV rhodopsin of Pieris rapae (Arikawa et al. 2005). The in situ hybridisation study of Awata et al. (2009) suggests that the UV cells are located in the position of the distal $\mathrm{R} 1,2$ photoreceptors of type I and type III ommatidia.

The $\mathrm{V}$ and $\mathrm{B}$ receptor classes

The vertical orientation of maximal polarisation sensitivity puts the $\mathrm{V}$ cells safely into the position of the $\mathrm{R} 1,2$ photoreceptors in the distal tier of type II ommatidia. The spectral 
sensitivity of the $\mathrm{V}$ receptor suggests the expression of a violet rhodopsin peaking at $\sim 420 \mathrm{~nm}$. This value is similar to the peak wavelength $425 \mathrm{~nm}$ of the violet rhodopsin of Pieris rapae, PrV (Arikawa et al. 2005). The alternative is that the $\mathrm{V}$ class expresses two violet/blue rhodopsins (CeV1 and CeV2; Awata et al. 2009), which then must have very similar absorption spectra. In this case, to modify the sensitivity of the violet rhodopsin-based receptor to that of bB cells, a very dense distal filter would be needed (see Fig. 5).

The V class has a somewhat lower spectral sensitivity in the short-wavelength limb than that predicted by the template (not shown). This may be caused by lateral screening of the $\beta$-peaks of the apposed R3,4 receptors (presumably $\mathrm{nG}$ in type II ommatidia) by the corneal facet lens acting as a UV filter, or the reduced sensitivity is due to electrical coupling (Matić 1983).

The spectral sensitivity of the $\mathrm{nB}$ receptor class strongly deviates from a rhodopsin template. Furthermore, the nB receptors have lower polarisation sensitivity than the other violet/blue rhodopsin-based classes. Curiously, the two cells with apparently reliably estimated PS axes are oriented at $\sim 90^{\circ}$. At present, we do not have an explanation for this deviation from the expected PS axis angle $0^{\circ}$. A heuristic fit estimated the peak sensitivity at $\sim 455 \mathrm{~nm}$, with a narrow bandwidth (FWHM) of $\sim 35 \mathrm{~nm}$, whilst the template fit to the LW limb of the sensitivity spectra estimated the rhodopsin peak to be at $\sim 420 \mathrm{~nm}$, which suggests that the $\mathrm{nB}$ class is based on the same opsin(s) as the V class. In Pieris rapae, the double-peaked blue receptor class $(\mathrm{dB})$, present only in the fluorescent type II ommatidia of males, is based on a single violet rhodopsin peaking at $425 \mathrm{~nm}$. Its spectral sensitivity peak is shifted to $460 \mathrm{~nm}$ due to screening by a violet-absorbing, fluorescing pigment (Arikawa et al. 2005). We hypothesise that in the fluorescent type I ommatidia of $C$. erate, a similar mechanism modifies $\mathrm{V}$ receptors into $\mathrm{nB}$ receptors. Under this assumption, we have calculated the difference between the log spectral sensitivities of the $\mathrm{V}$ and $\mathrm{nB}$ classes and thus estimated that the fluorescing pigment absorbs maximally at $\sim 420 \mathrm{~nm}$ (Fig. 9), in line with the excitation used to elicit fluorescence in our previous study (Arikawa et al. 2009).

$\mathrm{The} \mathrm{bBb}$ and $\mathrm{bBs}$ receptor classes are the most difficult to interpret. We fitted the rhodopsin template to the longwavelength limbs, above $500 \mathrm{~nm}$. The peak wavelength estimates from fitting both in the logarithmic and linear domain were in the range $453-461 \mathrm{~nm}$, similar to the $453 \mathrm{~nm}$ peak wavelength of the blue rhodopsin of Pieris rapae, PrB. The spectral sensitivity of the bB classes could in principle be explained with the violet rhodopsin peaking at $420 \mathrm{~nm}$, but this would imply the presence of an excessive filter in the distal tier (density $>2 \log$ units in the range

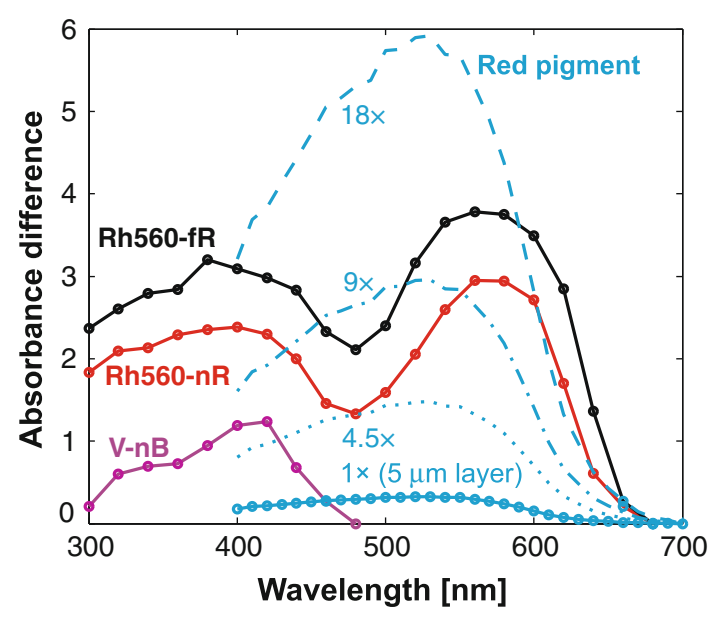

Fig. 9 Absorbance difference spectra. The difference between the log sensitivity spectra of the $\mathrm{nB}$ and $\mathrm{V}$ classes $(\mathrm{V}-\mathrm{nB})$ yields a spectrum peaking at $\sim 420 \mathrm{~nm}$ as the estimate of the absorbance spectrum of the fluorescing pigment (magenta line with circles). The absorbance difference spectra, calculated as the difference between the rhodopsin template (Rh560) and the spectral sensitivity profiles of $\mathrm{nR}($ red $)$ and $\mathrm{fR}$ (black) receptors have peaks $(\sim 3$ and $\sim 4 \log$ units, respectively) at around $560 \mathrm{~nm}$. The absorbance spectrum of a $5 \mu \mathrm{m}$ slab of the red pigment (blue line with circles) has been calculated from the thin layer transmittance spectrum measured by Arikawa et al. (2009) under the assumption that the red pigment has negligible absorption at $700 \mathrm{~nm}$. The cyan lines show a simulated effective optical thickness increase of 4.5-, 9- and 18-fold (dotted, dash-dotted and dashed blue lines, respectively). The latter factor has been chosen to fit the LW limb of the absorbance difference spectrum for the nR class (red) (colour figure online)

300-450 nm; see Fig. 5e), which seems to be unlikely). We hypothesise that the spectral shapes of the $\mathrm{bBb}$ and $\mathrm{bBs}$ classes are a consequence of lateral screening and the coexpression of the blue $\left(\lambda_{\mathrm{p}} \approx 460 \mathrm{~nm}\right)$ and the violet $\left(\lambda_{\mathrm{p}} \approx 420 \mathrm{~nm}\right.$ ) rhodopsin. The difference between the $\mathrm{bBb}$ and bBs spectral sensitivities can have various causes. First, assuming that bBs is located in type $\mathrm{I}$ and $\mathrm{bBb}$ in type II ommatidia, the difference could be due to spectral filtering by the fluorescing pigment. Second, the difference could be due to polymorphic expression of two violet/blue rhodopsins within the $C$. erate population. Third, R1,2 cells in type I and III ommatidia might contain both CeV1 and CeV2 mRNAs transcripts, but only one of them might be translated into opsin, e.g., via RNA interference. Fourth, the relative content of the two rhodopsins could differ amongst the photoreceptors, but this difference could not be detected with hybridisation (Awata et al. 2009). Simple modelling (not shown) suggests that if two rhodopsins are expressed in a single receptor in different mixing proportions, then the rhodopsin template fitting, as used here, would yield an estimate for $\lambda_{p}$ between the two rhodopsin peaks. In this case, the $\mathrm{nB}$ and $\mathrm{V}$ receptor classes could contain predominantly the violet rhodopsin, whilst $\mathrm{bBb}$ and $\mathrm{bBs}$ cells would contain a mixture of blue and violet rhodopsins. 
The $\mathrm{nG}$ and bG receptor classes

Fitting a rhodopsin template to the combined sensitivity spectra of the $\mathrm{bG}$ and $\mathrm{nG}$ classes yielded that the green rhodopsin $(\mathrm{CeL})$ has a peak wavelength at $559-565 \mathrm{~nm}$, corresponding well to the peak at $563 \mathrm{~nm}$ of the green rhodopsin of Pieris rapae (PrL; Wakakuwa et al. 2004). Both classes had a lower sensitivity in the short-wavelength range than the rhodopsin template $(25 \%)$. The reduction of UV sensitivity of the two receptor classes may be due to lateral screening by the UV and violet/blue rhodopsins in the apposed R1 and R2 cells and, in type I ommatidia, additionally due to screening by the distal fluorescent pigment.

The UV sensitivity of the $\mathrm{nG}$ class was $\sim 10 \%$, relative to the main peak. The difference between the green rhodopsin template and the measured sensitivity of $\mathrm{nG}$ class photoreceptors points to strong attenuation at $\sim 450 \mathrm{~nm}$ (Figs. 4, 5b). Consequently, nG had a narrower bandwidth $(\mathrm{FWHM} \approx 100 \mathrm{~nm})$ than the green rhodopsin template $(\mathrm{FWHM} \approx 110 \mathrm{~nm}$; Table 2). The attenuation is probably due to lateral screening by the blue rhodopsins $\left(\lambda_{\mathrm{p}} \approx 460 \mathrm{~nm}\right)$ of the R1 and R2 cells in type II ommatidia.

The cells of the bG class had an even lower UV sensitivity than those of the $\mathrm{nG}$ class. The bG cells are probably localised in type I and type II ommatidia. The absorbance difference spectrum between the bG sensitivity profile and the Rh560 template suggests $\sim 1 \log$ unit attenuation in the range 300-420 $\mathrm{nm}$ (not shown). The UV sensitivity of the $\mathrm{nG}$ and bG classes of $C$. erate seems to be more reduced than in the corresponding classes in Pieris rapae, where the L560 receptor class follows closely the rhodopsin template, whilst the L560-II class has about half a log unit reduced sensitivity between 350 and $450 \mathrm{~nm}$ (see Fig. 4a in Wakakuwa 2004).

Whilst the polarisation sensitivity of the $\mathrm{nG}$ class was very low, probably due to non-uniformly oriented microvilli, it was stronger in the bG class $(\psi \approx 0.2)$. The PS axis angle corresponds to the diagonal orientation $\left(\zeta \approx 45^{\circ}\right.$, $135^{\circ}$ ). This orientation is usually indicative of the proximal cells, but we favour the interpretation that most of the recorded bG and $\mathrm{nG}$ cells were actually the distal R3,4 receptors and that the diagonal orientation results from the curving of microvilli in the R3,4 rhabdomeres shown anatomically (Arikawa et al. 2009). We have compared the PS for the main and secondary (UV) peak of bG cells (Fig. 7). The PS axis angles, $\zeta$, were virtually identical, whilst the relative amplitudes, $\psi$, were in most cells slightly higher for the secondary peak. A similar phenomenon was observed in the double-peaked green photoreceptors R3,4 of Papilio xuthus (Bandai et al. 1992), where the higher PS of the secondary peak was explained by polarisation-dependent screening by neighbouring receptors $\mathrm{R} 1,2$. This explanation may be also valid for the $\mathrm{bG}$ and $\mathrm{R}$ cells of $C$. erate.
At present, we propose that the $\mathrm{nG}$ cells are $\mathrm{R} 3,4$ photoreceptors located in type II ommatidia and that the bG cells are those in type I and III ommatidia. Considering the variability in the data, we suspect that the classification into bG and $\mathrm{nG}$ receptors is somewhat arbitrary, as it seems likely that more subclasses could exist, for instance for the bG cells (bG1 and bG2 in Table 2). In this light, we mention the possibility that a few of the recorded $b G$ and $n G$ cells were actually proximal R5-8 photoreceptors in the dorsal part of the eye, where there is no perirhabdomal red pigment, so that the CeL-containing proximal R5-8 photoreceptors presumably retain a peak in the green (Arikawa et al. 2009). To conclusively identify all the green receptor classes, spectral sensitivity measurements should be combined with dye injection (Qiu and Arikawa 2003a).

The B and G receptors of the honeybee and other Hymenoptera have an almost unattenuated $\beta$-peak sensitivity and consequently the colour discrimination ability in the UV range is much lower than that at longer wavelengths (Backhaus 1991; Peitsch et al. 1991). It seems that $C$. erate has developed a mechanism that strongly reduces the $\beta$-peak sensitivity of the green receptors. This could potentially lead to an enhancement of colour discrimination in the UV wavelength range. A strong $\beta$-peak sensitivity reduction has also been measured in the single-peaked green class of Papilio xuthus (Bandai et al. 1992; Koshitaka et al. 2008). We note that the low UV sensitivity of the green receptors also opens up the possibility of using selective adaptation in the short-wavelength range as a tool to interpret extracellular recordings (Goldsmith 1986). Indeed, early extracellular recordings on a similar species from North America, C. eurytheme (Post and Goldsmith 1969), yielded a sensitivity peak at $\sim 560 \mathrm{~nm}$ whilst using selective UV adaptation. Interestingly, the red peaks in the American species were hinted to be at $\sim 600$ and $\sim 630 \mathrm{~nm}$, somewhat shorter than in our study.

\section{The $\mathrm{R}$ receptor classes}

The photoreceptors from the $\mathrm{R}$ class had a diagonally oriented PS, implying that they were located in the proximal tier of the eye. The PS axis angles for the main peak and secondary peak at $\sim 480 \mathrm{~nm}$ were very similar, whilst the relative polarisation sensitivity amplitudes were in most cells slightly higher for the secondary peak, similarly as in the bG receptors (Fig. 7). We individually fitted the eight red cells with symmetric double Gaussian functions (Fig. 10a). The linear estimates for the main peak wavelength, $\lambda_{1}$, were between 646 and $667 \mathrm{~nm}$, with narrow bandwidths (FWHM, $\delta_{1}$ ) of $25-50 \mathrm{~nm}$. The wavelength of the secondary peak, $\lambda_{2}$, was $\sim 490 \mathrm{~nm}$. We have noticed that the two cells with the lowest secondary peak amplitude $\left(a_{2} / a_{1}\right)$ had the left cutoff (WLHM, $\left.\lambda_{1}-\delta_{1} / 2\right)$ of the main 

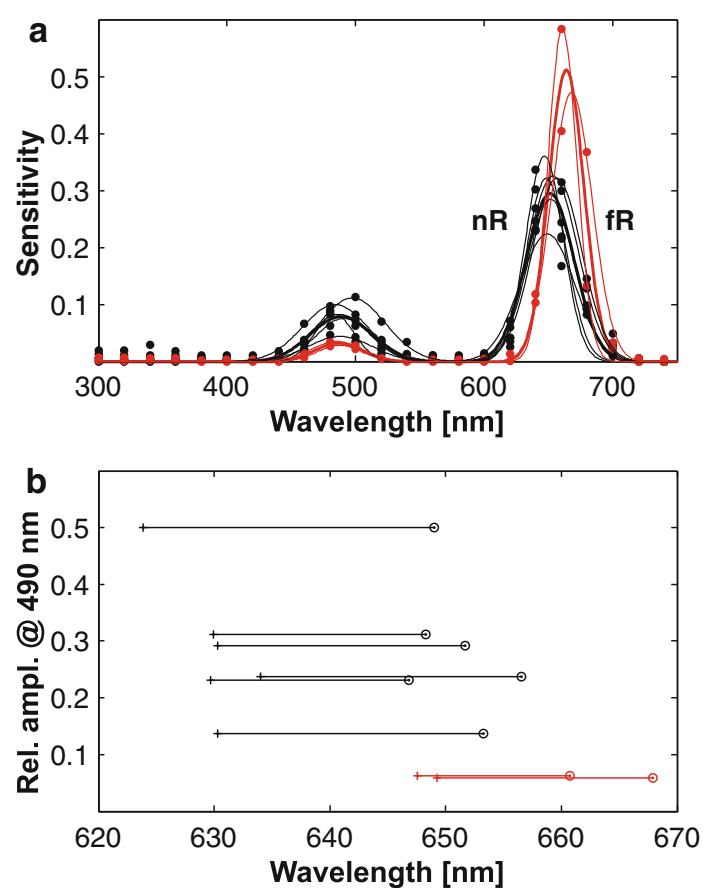

Fig. 10 Analysis of individual red receptors. a Fits of the sum of double Gaussian functions to the sensitivity profiles of the eight receptors from the red class with the provisional division into the $\mathrm{nR}$ subclass (black) and fR subclass (red). Dots show the experimental data. Thin lines show fits to individual cells and thick lines show the fits to the joined data from six $\mathrm{nR}$ cells (black) and two fR cells (red). b The relation between the peak position estimates (circles), the left cutoff estimates (WLHM; crosses) and the relative amplitude of the secondary peak at $480 \mathrm{~nm}$ ( $y$-axis) (colour figure online)

peak shifted farthest into the red. We have used this to support the division of red cells into the $\mathrm{nR}$ and $\mathrm{fR}$ classes. The estimated cutoff wavelengths (WLHM) were $\sim 630 \mathrm{~nm}$ for the $\mathrm{nR}$ and $\sim 650 \mathrm{~nm}$ for the $\mathrm{fR}$ class (Table 3 ). The main peak values of the $\mathrm{nR}$ and $\mathrm{fR}$ classes were at $\sim 650$ and $\sim 665 \mathrm{~nm}$, respectively.

The possibility for the existence of the separate $\mathrm{nR}$ and $\mathrm{fR}$ classes is corroborated by the eye shine measurements, which showed that, although there was only one type of red perirhabdomal pigment, the optical filtering was substantially stronger for the FR730 than for VR670 ommatidia (Arikawa et al. 2009). The alternative is that $C$. erate possesses a continuum of $\mathrm{R}$ receptors. In any case, our data indicate that the effective optical density of the perirhabdomal red pigment is variable. A larger number of spectral measurements from red receptors, with smaller steps between the stimulating wavelengths, will be necessary to substantiate the division into the $\mathrm{fR}$ and $\mathrm{nR}$ classes.

In comparison, Pieris rapae has two classes of proximally located long-wavelength receptors, L620 and L640, which are localised in the ommatidia with pale red and deep red perirhabdomal screening pigment, respectively. A blue secondary peak can also be seen in the spectral sensitivity curve of the L640 receptors of Pieris rapae (see Fig. 4b in Wakakuwa et al. 2004). The estimated peak wavelengths in $C$. erate $(\sim 650$ and $\sim 665 \mathrm{~nm})$ are more strongly redshifted compared to those of Pieris. To our knowledge, $C$. erate has the farthest red-shifted green receptors found in insects so far. For comparison, in the multi-spectral system of the mid-band in the eye of the mantis shrimp (stomatopod), Neogonadactylus oerstedii, the farthest red receptor peaks at $\sim 700 \mathrm{~nm}$ (Marshall et al. 2007).

Several components could contribute to the modification of the sensitivity spectrum of the proximal receptors. The most important absorber is the red screening pigment, whilst distal visual and screening pigments may also absorb significantly in the short-wavelength range (300-450 nm). Minor spectral contributions may also be due to corneal transmission, waveguide launching or tapetal reflection (Wakakuwa et al. 2004). We have calculated the effective rhabdom absorbance from the absorbance difference between the green rhodopsin template $\left(\lambda_{\mathrm{p}}=560 \mathrm{~nm}\right)$ and the spectral sensitivities of the $\mathrm{nR}$ and fR classes. The peak of the effective absorbance coincides with the green rhodopsin peak and suggests extremely efficient filtering ( 3 and $4 \log$ units of attenuation for the $\mathrm{nR}$ and $\mathrm{fR}$ class, respectively, over the pigment layer length of $\sim 300 \mu \mathrm{m})$. The effective absorbance in the blue range at $\sim 480 \mathrm{~nm}$ is less severe, $\sim 1.5$ to $\sim 2 \log$ units, resulting in the blue sensitivity peak of the red receptors. A similar, albeit less strong, rhabdom absorbance has been calculated for the deep red pigment containing L640 receptors of Pieris rapae, where the absorbance difference spectrum peaks of $\sim 2 \log$ units are at $420 \mathrm{~nm}$ and $580 \mathrm{~nm}$, whilst the absorbance difference dips at $\sim 1 \log$ unit at $480 \mathrm{~nm}$ (Wakakuwa et al. 2004).

The simulated thick-layer spectrum of the red pigment in C. erate was calculated on the basis of the transmittance spectrum of red pigment granules measured from $5 \mu \mathrm{m}$ thick tissue slabs (Arikawa et al. 2009). To match the longwavelength limb of the absorbance difference spectrum for the $\mathrm{nR}$ class receptors, we had to increase the effective optical thickness of the red pigment to about 18-fold (Fig. 9). We note that this factor should be considered with caution, because the assumption of full effective transmittance in the long-wavelength range (at $700 \mathrm{~nm}$ ) may not be fully valid. The light may be scattered out of the waveguide without absorption, due to refractive index difference between the red pigment granules and the surrounding medium. Still, the shapes of the simulated and measured effective absorbance spectra are clearly very different. We hypothesise that the effectiveness of the red screening pigment is diminished below $\sim 550 \mathrm{~nm}$, because at shorter wavelengths a smaller proportion of light energy travels outside the rhabdom where it can be absorbed by the pigment granules (Stavenga 2006). In the short-wavelength range (300-450 nm), effective 
absorption could be mostly due to distal screening by rhodopsins in R1-4 receptors and/or the fluorescing pigment. The combined effect is a dip in the effective absorbance spectrum, resulting in the secondary peak of red receptors at $\sim 480 \mathrm{~nm}$. Further modelling of the rhabdom will however be necessary to substantiate this hypothesis.

\section{Intensity-response characteristics}

The intensity-response characteristics of photoreceptors could be well described with a Hill sigmoid. The Hill slope parameter varied amongst the recorded cells, with a grand average for all cells of $h=0.73$. We found a weak negative correlation between the Hill slope parameter, $h$, and peak voltage, $V_{\mathrm{p}}$.

The rather broad range of the Hill slope (0.55-0.92) may be an artefact due to shunting, crosstalk from neighbouring receptors or ERG contamination (see Fig. 1), but it may also be an intrinsic property of the receptors (Heimonen et al. 2006). The latter possibility is supported by the finding that in the case of the $\mathrm{bG}$ receptor class, $\log R$ (the parameter describing the cell sensitivity) ranges over 1.3 $\log$ unit. We suggest that this variation is due to the intrinsic variation in cell properties, because the stimulus and recording conditions were always optimally adjusted and could hardly result in a 20 -fold difference in sensitivity. Different receptor classes with different contrast coding functions may maximise the information capacity of the retina and the visual system (e.g., UV receptors may have a steeper contrast for looking skywards), but as yet we have no firm evidence for this.

\section{The RWC method}

To estimate the effective intensity of light applied in the spectral and polarisation sensitivity scans, we compared the responses from the test run to the calibration run with a new method based on the RWC. The responses to a series of test stimuli were compared (using the least-squares approach) to an array of interpolated responses from the calibration run. The standard method for the analysis of pulse responses is to fit a Hill equation to the amplitudes of the responses from the calibration series. The response amplitudes are usually measured either as peak or plateau values; a small part of the trace where the signal is stationary is then averaged. In comparison, the RWC method offers two principal advantages. First, more independent information is extracted from the measurement without averaging, thus reducing the estimation noise. Second, the estimator errors of the modelled function are not back-propagated via the reverse transformation: a major problem with the standard method, especially near the threshold and saturation levels of the Hill sigmoid (Fig. 1b). Third, because there is no model assumed, there is no danger of using the wrong model. Fourth, the method can in principle also be used with bipolar or hyperpolarising stimuli, whilst the Hill sigmoid method can only deal with unipolar responses. The advantages of the RWC method can be appreciated from the better self-test result (Fig. 3).

The current implementation of the RWC method, using a polynomial for the interpolation, has two shortcomings. First, sometimes there may be a ripple in the flat parts of the intensity-response curves; this could be improved by using a different interpolation method, e.g., kernel filtering (Matić 1983). Second, the current implementation cannot reliably estimate effective intensities outside the calibrated range. This could be improved by extrapolating with a model function, but in practice it seems much easier to simply calibrate the whole range of interest. The method could be further improved by pre-filtering of the signals and by weighing the least-squares comparison based on the relative information content of individual samples. Another possible improvement that could estimate and correct for the contribution of the hyperpolarising crosstalk component (see Fig. 1) would be to measure a second intensityresponse curve in the hyperpolarising region and use nonlinear minimisation of the RMSD value in the second pass to find the best linear mixture of the two intensity-response curves.

\section{Comparison between Colias and Pieris}

The molecular and anatomical studies of $C$. erate (Awata et al. 2009; Arikawa et al. 2009) showed that $C$. erate employs four opsins, $\mathrm{CeUV}, \mathrm{CeV} 1, \mathrm{CeV} 2$ and $\mathrm{CeL}$, of which $\mathrm{CeV} 1$ and $\mathrm{CeV} 2$ were always co-expressed. Furthermore, a single red perirhabdomal screening pigment occurs in all ommatidia of the ventral area, and a distal, violetabsorbing fluorescing pigment exists in a subset of ommatidia. Of the three ommatidial types identified, the trapezoidal type (I) is fluorescing and has one cell expressing CeUV and one coexpressing $\mathrm{CeV} 1$ and $\mathrm{CeV} 2$, the square type (II) contains two photoreceptors coexpressing $\mathrm{CeV} 1$ and $\mathrm{CeV} 2$, and the rectangular type (III) contains two CeUV-based UV receptors. The opsin expression pattern is retained in the dorsal region, where the red pigment is absent (Awata et al. 2009). The situation is similar in Pieris rapae, which expresses PrUV, PrV, PrB and PrL in three types of ventral ommatidia, resulting in eight receptor classes altogether (Qiu and Arikawa 2003a; Wakakuwa et al. 2004; Arikawa et al. 2005; Stavenga and Arikawa 2006; Wakakuwa et al. 2007).

In $C$. erate, we have so far found nine receptor classes: one UV class, four violet/blue rhodopsin-based classes (V, $\mathrm{nB}, \mathrm{bBb}, \mathrm{bBs}$ ), two green classes ( $\mathrm{nG}$ and $\mathrm{bG}$ ) and two red classes (nR and fR). Table 4 and Fig. 11 summarise the 
Table 4 Proposed combinations of ventral ommatidial types

\begin{tabular}{|c|c|c|c|}
\hline Ventral type & I & II & III \\
\hline Fraction & 0.48 & 0.39 & 0.13 \\
\hline Geometry & Trapezoidal & Square & Rectangular \\
\hline Red pigment & Deep red & Deep red & Deep red \\
\hline Fluorescence & $\begin{array}{l}\text { Exc. }<420 \mathrm{~nm} \\
\text { Emission green }\end{array}$ & - & - \\
\hline Eye shine & VR670 & FR730 & FR730 \\
\hline $\mathrm{R} 1$ opsin & CeUV & $\mathrm{CeV} 1+\mathrm{CeV} 2$ & CeUV \\
\hline $\mathrm{R} 2$ opsin & $\mathrm{CeV} 1+\mathrm{CeV} 2$ & $\mathrm{CeV} 1+\mathrm{CeV} 2$ & CeUV \\
\hline R1 rhodopsin & 360 & $420(+460)$ & 360 \\
\hline R2 rhodopsin & $420(+460)$ & $420(+460)$ & 360 \\
\hline R1 class & UV' (?) & $\mathrm{V}, \mathrm{bBb}, \mathrm{bBs}$ & UV \\
\hline $\mathrm{R} 2$ class & $\mathrm{nB}, \mathrm{bBb}, \mathrm{bBs}$ & $\mathrm{V}, \mathrm{bBb}, \mathrm{bBs}$ & UV \\
\hline R3-8 opsin & $\mathrm{CeL}$ & $\mathrm{CeL}$ & $\mathrm{CeL}$ \\
\hline R3-8 rhodopsin & 560 & 560 & 560 \\
\hline R3-4 class & $\mathrm{bG}$ & $\mathrm{nG}$ & bG \\
\hline R5-8 class & $\mathrm{nR}$ & $\mathrm{fR}$ & $\mathrm{fR}$ \\
\hline
\end{tabular}

proposed ommatidial localisation of the photoreceptor classes in the ventral eye part. Taking the absorbance spectrum of the fluorescing pigment into consideration (Fig. 9), we suggest a possibility that type I ommatidia contain another UV rhodopsin-based receptor, which may have a reduced quantum efficiency and a reduced relative sensitivity in the violet range. At this point, due to lack of firm evidence, we also cannot unequivocally identify the localisation of the $\mathrm{bBb}$ and $\mathrm{bBs}$ classes. We also expect further subclassification of green receptors and possible additional receptor classes in the dorsal area of the eye.

The absorption spectrum of the red pigment of $C$. erate (Arikawa et al. 2009) is similar to that of the deep red pigment of Pieris rapae (unpublished). In both species, R1,2 receptors expressing violet/blue opsins have a larger rhabdom cross section than those expressing UV opsins. Another similarity is that in both species, the dorsal areas are devoid of perirhabdomal pigments, indicating that no photoreceptors in the dorsal area are especially tuned to red wavelengths, presumably because the sky does not have much useful information contained in the red wavelength band.

Interestingly, Colias and Pieris use different combinations of red and fluorescing pigments to implement additional photoreceptor classes. We note that in Anthocharis scolymus, a pierid species evolutionarily between the Colias erate and Pieris rapae (Braby et al. 2006), the fluorescing pigment is, as in Colias, expressed in trapezoidal ommatidia (Takemura et al. 2007). It also seems that the red pigment found in Anthocharis is similar to that of Colias and the deep red pigment of Pieris. Since Coliadinae are primordial to Pierinae (Braby et al. 2006), it could be that the pale red pigment is a recent evolutionary develop-
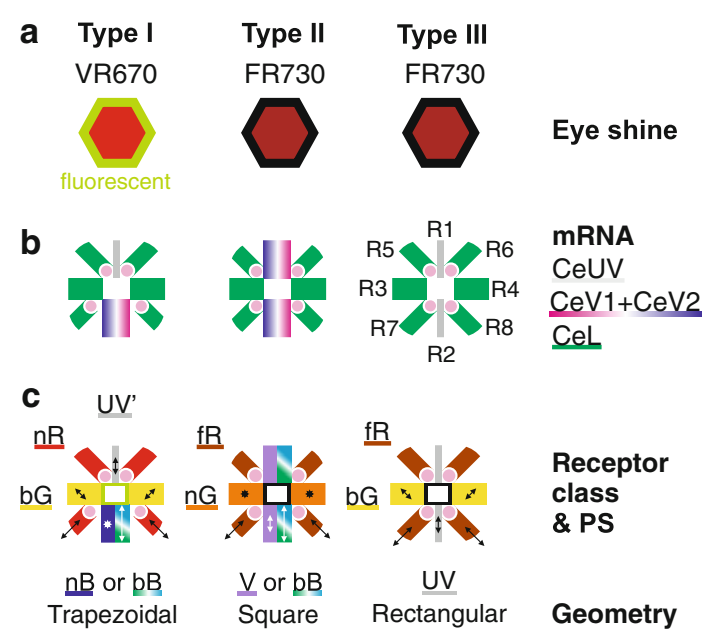

Fig. 11 Provisional scheme of the three proposed ventral ommatidial types in $C$. erate. a Only Type I ommatidia fluoresce under violet $(<420 \mathrm{~nm})$ excitation. b CeV1 and CeV2 mRNA are co-expressed in R1 and R2 cells of Type I and II ommatidia (Awata et al. 2009). Receptor R9 (always expressing CeL) has been omitted for the sake of clarity. c The size and orientation of arrows depicts the polarisation sensitivity (PS) of the various receptor classes. The relative size of the coloured slabs depicts how much microvilli each receptor class contributes to the rhabdom. Colour coding is consistent with receptor classes in Fig. 4 (colour figure online)

ment. In this case, however, it remains unclear, why a second, paler pigment would evolve to cause a smaller red shift, whilst the same effect can also be achieved by reducing the effective optical thickness of the deep red pigment, as is the case in Colias $\mathrm{nR}$ and $\mathrm{fR}$ receptor classes.

\section{General discussion}

Butterflies use gene duplication and rhabdomal filtering as the two main mechanisms for expanding their photoreceptor sets. Opsin gene duplication has been shown in most butterfly families: violet/blue opsin duplication has been found in Pieridae and Lycaenidae, UV opsin duplication in Nymphalidae and green opsin duplication in Papilionidae and Riodinidae. Pierid butterflies seem to use filtering as the main strategy of photoreceptor set expansion into the red wavelength range, whilst papilionids combine filtering with opsin duplication (Arikawa et al. 2005, 2009; Sison-Mangus et al. 2006; Briscoe 2008, Awata et al. 2009; Briscoe et al. 2010). On the other hand, retention of the basic trichromatic plan has been electrophysiologically demonstrated in some species of the nymphalid subfamily Nymphalinae (Kinoshita et al. 1997), where some (but not all) species have a uniform eye shine that hints at the absence of red screening pigments (Stavenga 2002a; Briscoe and Bernard 2005). Red screening pigments may also be missing in some members of the nymphalid subfamiliy Satyrinae (unpublished), but they are present in the closely related subfamily Heliconiinae (Zaccardi et al. 2006). Judging from the heterogeneity of the eye shine 
(Stavenga et al. 2001; Stavenga 2002a, b), it is quite likely that a majority of species belonging to different butterfly families have an expanded set of photoreceptors in the longwavelength range.

In this study, we have shown that Colias erate possesses an expanded set of long-wavelength receptors, with their sensitivity maxima between 645 and $670 \mathrm{~nm}$. The advantage of having additional red colour channels remains an intriguing, unanswered question. Thus far, it has been shown that the red receptor in Papilio xuthus is used for tetrachromatic colour vision in foraging behaviour (Koshitaka et al. 2008). A pioneering study of colour-specific behaviours of Pieris brassicae, which presumably has similar red receptor classes as $P$. rapae, has shown that the feeding response behaviour is elicited by monochromatic stimuli in the blue-green range $(420-520 \mathrm{~nm})$ and in a narrow red range (590-610 nm), hinting at a green-red opponency mechanism (Scherer and Kolb 1987). A similar mechanism may also exist in Colias erate. Possibly, the red receptors of $C$. erate, having their sensitivity peaks close to the red absorption maximum of chlorophyll, enhance the visibility of conspecifics and the contrast of flowers against the foliage. Another possibility would be that the red receptors are used for the detection of the host plant or its quality. Indeed, red receptors have been implied to play a role in oviposition behaviour, and it has been suggested that those insects that are interested in foliage (e.g., butterflies, some hymenopterans), may benefit from having red receptors (Kelber 1999). Yet, the evolutionary drive that pushes the red sensitivity of $C$. erate further away from the rhodopsin peak remains unexplained.

We conclude that further comparative studies of butterfly physiological optics (eye shine and electrophysiology), supported by molecular biology and anatomy, will provide important insights into the evolution of this diverse insect group, especially when the findings will be related to visual ecology and behaviour, with the focus on colour contrasts between the wings, flowers, host plants and the environmental background.

Acknowledgments The financing of this work was provided by AFOSR/EOARD grant no. FA8655-08-1-3012 and JSPS grant 21247009. We thank Gregor Belušič, Eric J. Warrant and the reviewers for valuable comments on the manuscript.

Open Access This article is distributed under the terms of the Creative Commons Attribution Noncommercial License which permits any noncommercial use, distribution, and reproduction in any medium, provided the original author(s) and source are credited.

\section{References}

Arikawa K (1999) Color vision. In: Eguchi E, Tominaga Y (eds) Atlas of arthropod sensory receptors. Springer-Verlag, Tokyo, pp 23-32
Arikawa K (2003) Spectral organization of the eye of a butterfly, Papilio. J Comp Physiol A 189:791-800

Arikawa K, Stavenga DG (1997) Random array of colour filters in the eyes of butterflies. J Exp Biol 200:2501-2506

Arikawa K, Uchiyama H (1996) Red receptors dominate the proximal tier of the retina in the butterfly Papilio xuthus. J Comp Physiol A 178:55-61

Arikawa K, Inokuma K, Eguchi E (1987) Pentachromatic visual system in a butterfly. Naturwissenschaften 74:297-298

Arikawa K, Wakakuwa M, Qiu X, Kurasawa M, Stavenga DG (2005) Sexual dimorphism of short-wavelength photoreceptors in the small white butterfly, Pieris rapae crucivora. J Neurosci 25:5935-5942

Arikawa K, Pirih P, Stavenga DG (2009) Rhabdom constriction enhances filtering by the red screening pigment in the eye of the Eastern Pale Clouded yellow butterfly, Colias erate (Pieridae). J Exp Biol 212:2057-2064

Awata H, Wakakuwa M, Arikawa K (2009) Evolution of color vision in pierid butterflies: blue opsin duplication, ommatidial heterogeneity and eye regionalisation in Colias erate. J Comp Physiol A 195:401-408

Backhaus WGK (1991) Color opponent coding in the visual system of the honeybee. Vis Res 31:1381-1397

Bandai K, Arikawa K, Eguchi E (1992) Localization of spectral receptors in the ommatidium of butterfly compound eye determined by polarization sensitivity. J Comp Physiol A 171:289-297

Braby MF, Vila R, Pierce NE (2006) Molecular phylogeny and systematics of the Pieridae (Lepidoptera: Papilionoidea): higher classification and biogeography. Zool J Linn Soc 147:239-275

Briscoe AD (2008) Reconstructing the ancestral butterfly eye: focus on the opsins. J Exp Biol 211:1805-1813

Briscoe AD, Bernard GD (2005) Eyeshine and spectral tuning of long wavelength-sensitive rhodopsins: no evidence for red-sensitive photoreceptors among five Nymphalini butterfly species. J Exp Biol 208:687-696

Briscoe AD, Chittka L (2001) The evolution of color vision in insects. Annu Rev Entomol 46:471-510

Briscoe AD, Bybee SM, Bernard GD, Yuan F, Sison-Mangus MP, Reed RD, Warren AD, Llorente-Bousquets J, Chiao CC (2010) Positive selection of a duplicated UV-sensitive visual pigment coincides with wing pigment evolution in Heliconius butterflies. Proc Natl Acad Sci USA 107:3628-3633

Goldsmith TH (1986) Interpreting trans-retinal recordings of spectral sensitivity. J Comp Physiol A 159:481-487

Govardovskii VI, Fyhrquist N, Reuter T, Kuzmin DG, Donner K (2000) In search of the visual pigment template. Vis Neurosci 17:509-528

Hamdorf K (1979) The physiology of invertebrate visual pigments. In: Autrum H (ed) Handb Sens Physiol, vol VII/6A. Springer, Berlin, pp 145-224

Heimonen K, Salmela I, Kontiokari P, Weckström M (2006) Large functional variability in cockroach photoreceptors: optimization to low light levels. J Neurosci 26:13454-13462

Kelber A (1999) Ovipositing butterflies use a red receptor to see green. J Exp Biol 202:2619-2630

Kinoshita M, Sato M, Arikawa K (1997) Spectral receptors of nymphalid butterflies. Naturwissenschaften 84:199-201

Kitamoto J, Ozaki K, Arikawa K (2000) Ultraviolet receptors and violet receptors express identical mRNA encoding an ultraviolet-absorbing opsin: identification and histological localization of two mRNAs encoding short wavelength-absorbing opsins in the retina of the butterfly Papilio xuthus. J Exp Biol 203:28872894

Koshitaka H, Kinoshita M, Vorobyev M, Arikawa K (2008) Tetrachromacy in a butterfly that has eight varieties of spectral receptors. Proc R Soc B 275:947-954 
Land MF, Nilsson D-E (2002) Animal eyes. Oxford University Press, Oxford

Laughlin SB (1981) Neural principles in the peripheral visual systems of invertebrates. In: Autrum H (ed) Handb Sens Physiol, vol VII/ 6B. Springer, Berlin, pp 135-280

Marshall J, Cronin TW, Kleinlogel S (2007) Stomatopod eye structure and function: a review. Arthr Struct Devel 36:420-448

Matić T (1983) Electrical inhibition in the retina of the butterfly retina. J Comp Physiol A 152:169-182

Menzel R, Backhaus WGK (1989) Color vision of honey bees: phenomena and physiological mechanisms. In: Stavenga DG, Hardie RC (eds) Facets of vision. Springer, Berlin, pp 281-297

Miller WH, Bernard GD (1968) Butterfly glow. J Ultrastruct Res 24:286-294

Peitsch D, Fietz A, Hertel H, de Souza J, Fix Ventura D, Menzel R (1991) The spectral input systems of hymenopteran insects and their receptor-based colour vision. J Comp Physiol A 170:23-40

Post CT Jr, Goldsmith TH (1969) Physiological evidence for color receptors in the eye of a butterfly. Ann Ent Soc Am 62:1497-1498

Qiu X, Arikawa K (2003a) The photoreceptor localization confirms the spectral heterogeneity of ommatidia in the male small white butterfly, Pieris rapae crucivora. J Comp Physiol A 189:81-88

Qiu X, Arikawa K (2003b) Polymorphism of red receptors: sensitivity spectra of proximal photoreceptors in the small white butterfly Pieris rapae crucivora. J Exp Biol 206:2787-2793

Qiu X, Vanhoutte KJA, Stavenga DG, Arikawa K (2002) Ommatidial heterogeneity in the compound eye of the male small white, Pieris rapae crucivora. Cell Tissue Res 307:371-379

Ribi WA (1978) Ultrastructure and migration of screening pigments in the retina of Pieris rapae L. (Lepidoptera Pieridae). Cell Tissue Res 191:57-73

Scherer C, Kolb G (1987) Behavioral experiments on the visual processing of color stimuli in Pieris brassicae L (Lepidoptera). J Comp Physiol A 160:645-656

Sison-Mangus MP, Bernard GD, Lampel J, Briscoe AD (2006) Beauty in the eye of the beholder: the two blue opsins of lycaenid butter- flies and the opsin gene-driven evolution of sexually dimorphic eyes. J Exp Biol 209:3079-3090

Stavenga DG (2002a) Colour in the eyes of insects. J Comp Physiol A 188:337-348

Stavenga DG (2002b) Reflections on colourful butterfly eyes. J Exp Biol 205:1077-1085

Stavenga DG (2006) Invertebrate photoreceptor optics. In: Warrant EJ, Nilsson DE (eds) Invertebrate Vision. Cambridge University Press, Cambridge, pp 1-44

Stavenga DG, Arikawa K (2006) Evolution of color and vision of butterflies. Arthr Struct Dev 35:307-318

Stavenga DG, Kinoshita M, Yang EC, Arikawa K (2001) Retinal regionalization and heterogeneity of butterfly eyes. Naturwissenschaften 88:477-481

Takemura SY, Stavenga DG, Arikawa K (2007) Absence of eye shine and tapetum in the heterogeneous eye of Anthocharis butterflies (Pieridae). J Exp Biol 210:3075-3081

Vanhoutte KJA, Michielsen KFL, Stavenga DG (2003) Analyzing the reflections from single ommatidia in the butterfly compound eye with Voronoi diagrams. J Neurosci Meth 131:195-203

Wakakuwa M, Stavenga DG, Arikawa K (2007) Spectral organization of ommatidia in flower-visiting insects. Photochem Photobiol $83: 27-34$

Wakakuwa M, Stavenga DG, Kurasawa M, Arikawa K (2004) A unique visual pigment expressed in green, red and deep-red receptors in the eye of the small white butterfly, Pieris rapae crucivora. J Exp Biol 207:2803-2810

White RH, Xu H, Münch T, Bennett RR, Grable EA (2003) The retina of Manduca sexta: rhodopsin-expression, the mosaic of bluegreen and UV-sensitive photoreceptors. J Exp Biol 206:33373348

Zaccardi G, Kelber A, Sison-Mangus MP, Briscoe AD (2006) Color discrimination in the red range with only one long-wavelength sensitive opsin. J Exp Biol 209:1944-1955 